\title{
Bases de diseño, modelización, cálculo y comportamiento del viaducto de Almonte
}

\section{Design basis, structural modelling and detailed analysis of Almonte Viaduct}

\author{
Guillermo Capellán Miguel a, Emilio Merino Rasillo ${ }^{\mathrm{b}}$, Ysabel Guil Celada ${ }^{\mathrm{b}}$, \\ Javier Martínez Aparicio ${ }^{\mathrm{b}}$, y Pascual García Arias ${ }^{\mathrm{c}}$ \\ ${ }^{a}$ Dr. Ingeniero de Caminos, Canales y Puertos. Arenas \& Asociados, Santander, España \\ ${ }^{b}$ Ingeniero de Caminos, Canales y Puertos. Arenas \& Asociados, Madrid, España \\ cIngeniero de Caminos, Canales y Puertos. Idom Ingeniería, Madrid, España
}

Recibido el 23 de febrero de 2018; aceptado el 22 de febrero de 2019

\begin{abstract}
RESUMEN
El proyecto y construcción del viaducto de Almonte ha supuesto un reto por las excepcionales dimensiones del arco, que supera con su luz de $384 \mathrm{~m}$ cualquier experiencia previa en puentes arco ferroviarios. El diseño detallado de la estructura ha requerido desarrollar complejos modelos de cálculo, considerando la no linealidad geométrica y de materiales, así como todas las etapas del proceso constructivo. Las consideraciones de diseño específicas de los viaductos de alta velocidad han obligado a realizar comprobaciones adicionales que exceden el ámbito de la normativa actual. Se recoge en este artículo distintos aspectos del diseño conceptual y comportamiento de la estructura que por su interés resultan relevantes.
\end{abstract}

(C) 2020 Asociación Española de Ingeniería Estructural (ACHE). Publicado por Cinter Divulgación Técnica S.L.L. Todos los derechos reservados. PALABRAS CLAVE: arco; hormigón; bases de cálculo; modelización; funicularidad; cálculo no lineal.

\section{ABSTRACT}

The design and construction of the Almonte Viaduct has been a challenge due to the exceptional dimensions of the arch, which exceeds due to the $384 \mathrm{~m}$ span all previous experience in railway arch bridges. The detailed design of the structure has required the development of complex calculation models, considering both geometrical and material non-linearity, as well as all the stages of the construction process. The specific design considerations of the high-speed railway viaducts have forced to perform additional checks that exceed the scope of the current regulations. This article includes different aspects of the conceptual and detailed design of the structure that are relevant for their interest.

(C) 2020 Asociación Española de Ingeniería Estructural (ACHE). Published by Cinter Divulgación Técnica S.L.L. All rights reserved.

KEYWORDS: arch; concrete; design basis; structural modelling; funicularity; non-linear analysis.

1.

\section{INTRODUCCIÓN}

El puente sobre el río Almonte en el embalse de Alcántara es un puente arco con una luz principal de $384 \mathrm{~m}$ y una longitud total de $996 \mathrm{~m}$. Su diseño y construcción incluyen muchas características especiales y demandan unos análisis complejos para los cuales han debido ponerse a punto métodos de cálculo no necesarios en el análisis de estructuras convencionales, donde los fenómenos no lineales y efectos aeroelásticos resultan poco importantes, pero que en este caso son determinantes.
2.

\section{PARTICULARIDADES DE LA ESTRUCTURA}

Las particularidades que se dan en un puente para ferrocarril de alta velocidad son unas cargas de tráfico mucho mayores que las de carretera, unos efectos dinámicos por el paso de los convoyes, unas cargas horizontales notables y un trabajo de fatiga que no se puede despreciar. Todo esto debe conjugarse con unas consideraciones funcionales muy estrictas para que el nivel de servicio de la estructura no deba limitarse en ningún momento: deflexiones y aceleraciones admisibles pequeñas y una longitud entre juntas de dilatación de la vía limitada por motivos tecnológicos. Dadas esas particularidades, las luces mayores de $100 \mathrm{~m}$ hasta ahora han 


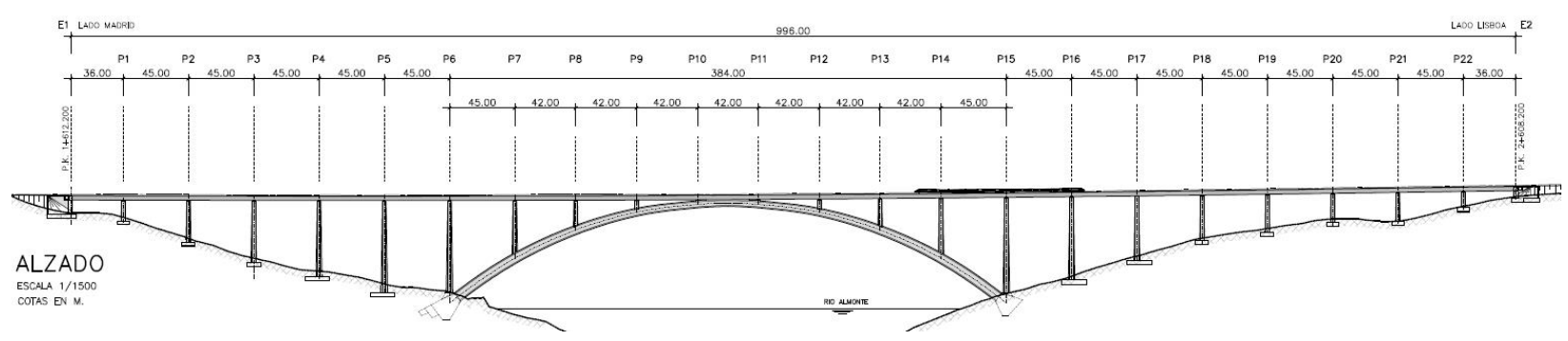

Figura 1. Alzado viaducto de Almonte.

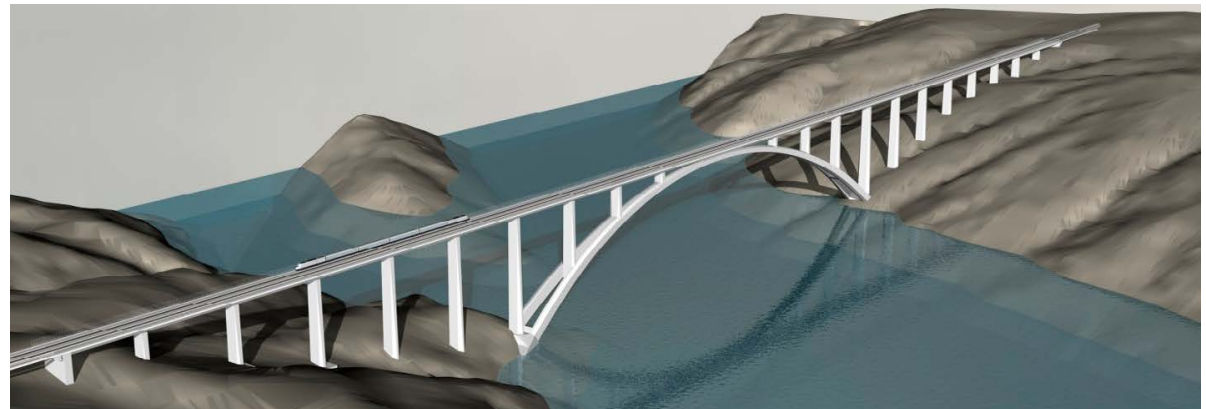

Figura 2. Imagen virtual correspondiente al estudio de tipologías del proyecto del viaducto de Almonte.

sido inusuales en los puentes para ferrocarril de alta velocidad y, por tanto, la luz que ha sido necesario adoptar para salvar el embalse de Almonte entra dentro de un rango excepcional.

A pesar de la singularidad del vano principal, este puente se conforma con un tablero típico con luces máximas de 45 m y 3.10 m de canto (relación canto/luz 1/14.5) a lo largo de sus $996 \mathrm{~m}$ (figuras 1 y 2). Esas luces se construyen por medio del conocido sistema de cimbra autolanzable, por lo que, es en el elemento arco y su zona de influencia estructural, donde se han debido poner en práctica métodos constructivos más elaborados; en concreto, el arco se ejecuta por medio de un carro de avance y un atirantamiento provisional desde dos torres metálicas provisionales situadas sobre el tablero en la vertical de las pilas de arranque del arco.

A consecuencia de la naturaleza de la propia estructura, de las condiciones de servicio, y del método constructivo, el análisis estructural debe incluir:

- Estudio de la directriz óptima del arco en base al cálculo de la directriz antifunicular de las cargas.

- Análisis detallado de las fases constructivas.

- Valoración del estado límite de servicio y último con modelos de cálculo no lineales.

- Análisis dinámico detallado.

- Entre los estudios especiales se ha de señalar la confirmación de los resultados de los estudios analíticos realizados del comportamiento de la estructura frente a las cargas de viento, mediante la realización de estudios experimentales con ensayo en túnel de viento.

En las páginas siguientes se muestran los puntos más significativos de algunos de estos cálculos, centrando las explicaciones en los aspectos relacionados con la forma de la directriz del arco, el cálculo no lineal de los estados límite último y de Servicio y la deformabilidad de la estructura.

La particularidad de la estructura también radica en los hormigones utilizados, habiéndose requerido para las dovelas del arco un hormigón autocompactable de alta resistencia HA80 , siendo este punto objeto de otro artículo del monográfico.
3.

\section{DISEÑO ESTRUCTURAL DE LA SOLUCIÓN}

\subsection{Influencia del encaje de la directriz del arco en el diseño del mismo}

En el diseño de puentes arco la búsqueda de la geometría de su directriz es un punto clave del diseño, ya que si es la adecuada trabajará por forma, es decir, sometido solo a tensiones normales de compresión en todos y cada uno de sus puntos, funcionando como antifunicular de las cargas. De esta forma, el material constituyente del arco estará exento de tracciones y, al no fisurar el hormigón, se garantiza la durabilidad del puente con un mantenimiento mínimo además de economizar en las cuantías de acero que son las que, dado el caso, deben resistir las tracciones.

En el caso de los puentes de ferrocarril de alta velocidad, la búsqueda de la directriz antifunicular impone una nueva realidad en la metodología empleada hasta la fecha en el encaje de la misma: en el ferrocarril los trenes de carga correspondientes al material móvil inducen en el arco esfuerzos de flexión importantes, siendo el efecto de la sobrecarga con respecto a las cargas permanentes mucho mayor, y por tanto deben ser considerados para el estudio de la directriz del arco.

Se convierte por ese motivo en pieza importante del diseño de un puente arco la decisión de si se acepta fisuración en el arco en situaciones de servicio o no. Se da el caso particular de que aceptar una fisuración limitada del arco en ciertas situaciones de servicio, hace difícil y encarece el cumplimiento de las deformaciones límites para el servicio de la estructura, ya que las fisuras degradan la rigidez del arco. Es decir, cuando esta degradación de la rigidez ocurre en un arco, los movimientos con los que responde a las cargas son mayores, y para lograr unas flechas admisibles adecuadas al uso ferroviario sería necesario dimensionar el puente para las inercias en fisuración, es decir, sería necesario aumentar la cantidad de material y el canto en comparación a si se pudiese evitar la fisuración. Es por eso que en los puentes arco de ferrocarril es aconsejable 


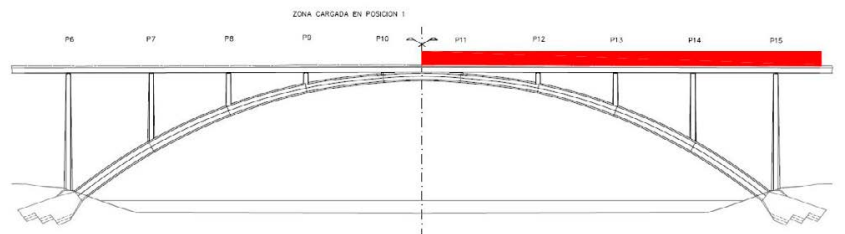

Figura 3. Posición 1 del tren en el estudio de la línea de presiones.

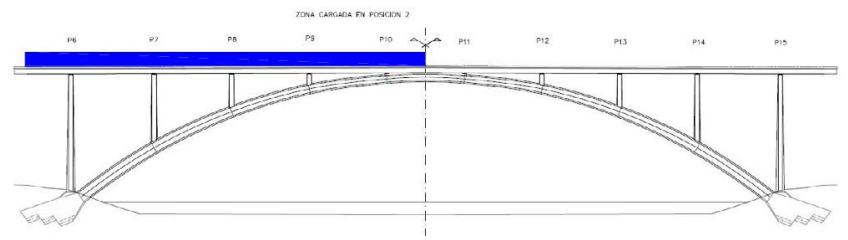

Figura 4. Posición 2 del tren en el estudio de la línea de presiones.

un criterio general de evitar la fisuración ante sobrecargas en situaciones de servicio aprovechando las herramientas en juego.

Para las situaciones de estado límite último (ELU), resulta prácticamente inevitable la fisuración con las solicitaciones producidas por las cargas mayoradas y las resistencias de los materiales minoradas, por lo que todas las comprobaciones referidas del comportamiento global del arco y la estabilidad del mismo en situaciones de ELU deben hacerse teniendo en cuenta los efectos de la fisuración.

Una vez establecida la influencia de la fisuración en el arco y definida su directriz como dato impuesto, el diseño va a estar presidido por la selección más adecuada del canto o, dicho en otros términos, de su rigidez a la flexión principal, es decir, aquella que controla las curvaturas para momentos flectores contenidos en el plano del arco.

El estudio de la rigidez principal se relaciona con la determinación de las posibles líneas de empuje a las que la estructura se somete y responde por su forma. El carácter antifunicular se refiere siempre a un sistema de cargas determinado. Para cualquier otro sistema de cargas que actúe sobre el arco se producirán en general flexiones. En el diseño estructural se hace necesario considerar todas las hipótesis de carga que se derivan del carácter variable de las sobrecargas. El procedimiento es simple: para cada configuración de cargas concreta es posible hallar la línea de empujes y ajustar una rigidez principal, es decir, el canto del arco ya que tanto la forma básica como el material del arco están definidos, con objeto de que la línea de presiones esté contenida en el núcleo central de las diferentes secciones [1].

La complejidad reside en que la sobrecarga principal son los trenes, que circulan sobre el puente, pueden ser de varios tipos y pueden estar o no presentes en distintos grados por cualquiera de las vías que se asientan en el tablero y en cualquiera de sus puntos. Por tanto el tratamiento debe ser idéntico al de una envolvente. La directriz más apropiada estará dentro de la superficie delimitada por el haz de funiculares.

Para el ajuste de la directriz se determinaron las líneas de fuerza en el arco para diferentes hipótesis de carga teniendo en cuenta el peso propio, las cargas permanentes, variaciones térmicas y paso simultáneo de dos trenes. Los trenes considerados incluyen tanto los trenes HSLM-A como los trenes reales de alta velocidad recogidos en la IAPF. Para tener en cuenta los efectos dinámicos de las cargas móviles, se emplean las cargas estáticas con el coeficiente de impacto por el efecto dinámico de las cargas correspondientes a la velocidad crítica.
Para cada uno de los trenes se estudiaron varias posiciones, para tener en cuenta tanto momentos flectores positivos como negativos. Se indican dos de las posiciones estudiadas (figuras 3 y 4).

A continuación, se muestran las gráficas correspondientes al núcleo central y las líneas de fuerza para las cargas permanentes y las combinaciones con el paso dinámico de los trenes HSLM-Al y AVE a una velocidad de $330 \mathrm{~km} / \mathrm{h}$ (por ser esta la velocidad a la que se producen los mayores efectos dinámicos). (figuras 5, 6 y 7).

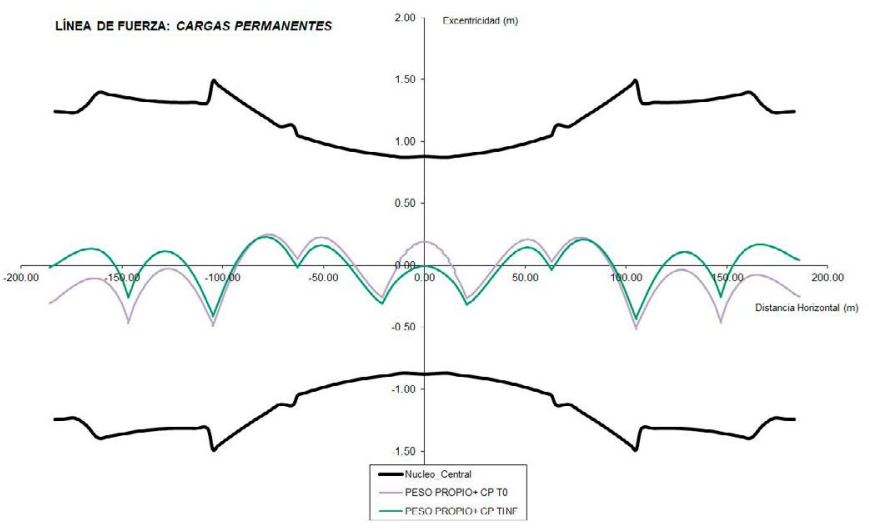

Figura 5. Núcleo central y líneas de fuerza para cargas permanentes.

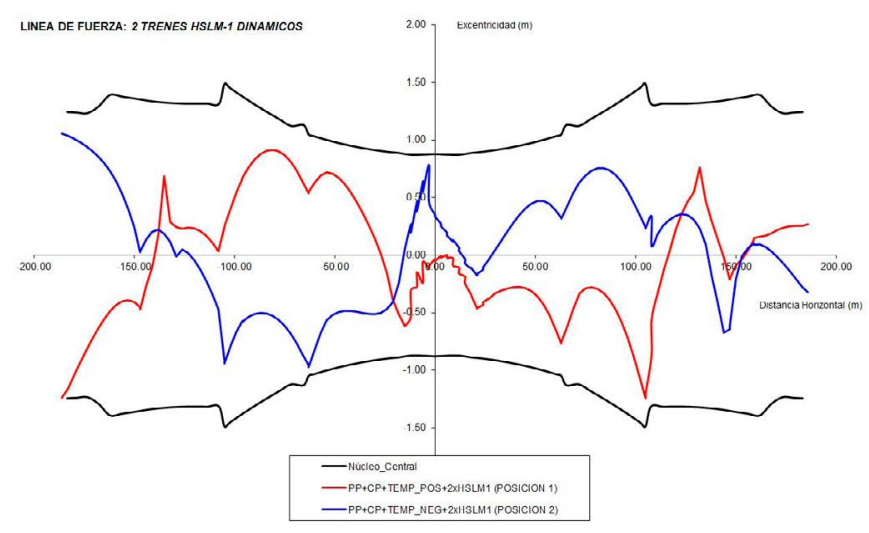

Figura 6. Núcleo central y líneas de fuerza para el paso de 2 trenes HSLM-1 a $330 \mathrm{~km} / \mathrm{h}$.

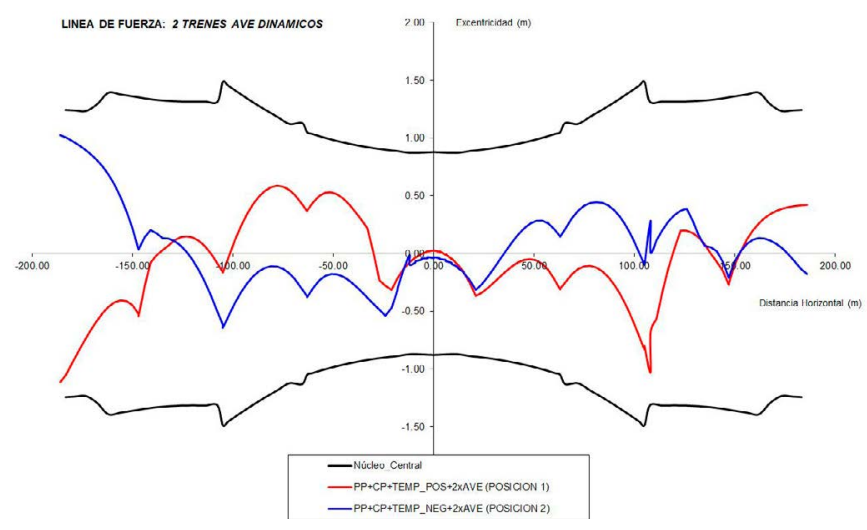

Figura 7. Núcleo central y líneas de fuerza para el paso de 2 trenes AVE a $330 \mathrm{~km} / \mathrm{h}$.

En la figura siguiente se muestra un alzado de un semiarco del viaducto en donde se puede visualizar el encaje de las líneas de presiones envolventes en el paso dinámico de un tipo 
de tren concreto dentro del núcleo central del arco con lo que se asegura la ausencia completa de fisuración en las condiciones de servicio (figuras 8 y 9).

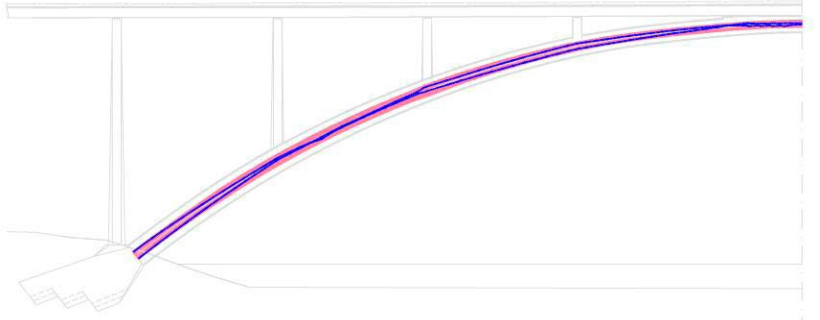

Figura 8. Núcleo central en rojo y línea de presiones en azul correspondiente a trenes HSLM-1 en paso dinámico a $320 \mathrm{~km} / \mathrm{h}$.

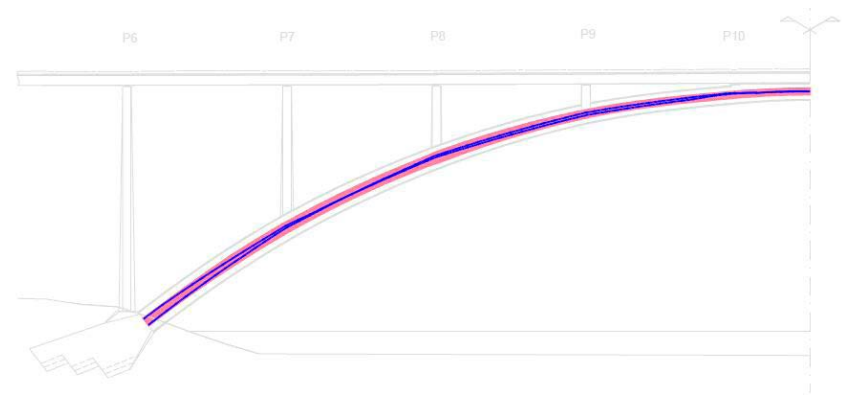

Figura 9. Núcleo central en rojo y línea de presiones en azul correspondiente a trenes AVE en paso dinámico a $320 \mathrm{~km} / \mathrm{h}$.

\subsection{Procedimiento constructivo}

El puente diseñado surge como resultado de un análisis multicriterio realizado sobre diferentes alternativas tipológicas, por ser la solución más ventajosa desde el punto de vista de la durabilidad, mantenimiento, del comportamiento ante cargas dinámicas y de confort y funcionalidad por la pequeña deformabilidad de la estructura frente a otras soluciones [2].

Una vez decidido el empleo del arco de hormigón, durante la fase de redacción del proyecto se estudiaron diferentes alternativas constructivas [3]:

- Construcción del arco de manera exenta con atirantamiento provisional, y con la ayuda de unas torres provisionales que se sitúan sobre el tablero en la vertical de las pilas de arranque del arco. En este caso las pilas y el tablero se ejecutan tras la finalización del arco.

- Construcción del arco de manera exenta con atirantamiento provisional y torres provisionales, realizando la parte central del arco mediante la flotación e izado de un tramo central en celosía metálica que sirve de cimbra para el posterior hormigonado de esta parte del arco. Este procedimiento puede considerarse como una variante del anterior.

- Construcción conjunta del arco con las pilas y el tablero, disponiendo diagonales provisionales entre arco y tablero, de tal manera que se configura durante las fases en voladizo una celosía de canto variable, actuando el tablero como cordón superior traccionado que se ancla al terreno.

- Construcción conjunta del arco con las pilas, disponiendo diagonales y un tirante provisional superior para conformar una celosía de canto variable. En este sistema constructivo, a diferencia del anterior, el tiro de tracción correspondiente al cordón superior de la celosía en voladizo no es recogido por el tablero, sino por un elemento provisional. En este caso el tablero se ejecuta tras la finalización del arco.

Durante la redacción del estudio de tipologías se descartaron las soluciones con tablero mixto, con lo cual la opción de construcción del arco con el sistema de diagonales que utilizan el tablero como cordón superior traccionado de una celosía no resulta conveniente, pues la tracción que se genera en el tablero no puede ser asumida de manera razonable por un tablero de hormigón pretensado.

Una vez analizadas todas las alternativas, y debido a los distintos condicionantes, se seleccionó el sistema de construcción del arco con avance en voladizo y torres metálicas provisionales como el más apropiado para este caso, ejecutando posteriormente las pilastras y el tablero mediante la ayuda de sendas autocimbras que avanzan desde ambos lados del arco apoyándose sobre el arco ya construido.

Aunque esta alternativa necesita más medios auxiliares que otras, presenta la ventaja de permitir un preciso control geométrico durante todas las etapas del proceso constructivo. Además, la presencia de los tirantes permite presolicitar al arco de forma completamente controlada con el objeto de conseguir le ley de flectores deseada con el puente cerrado sin necesidad de realizar una apertura en clave del arco previo al hormigonado de la dovela de cierre.

Como es sabido, la operación de apertura en clave de un arco tiene como objeto introducir un esfuerzo axil y un momento flector en la clave del arco para compensar el acortamiento elástico del hormigón y los efectos de retracción y fluencia que se producirán una vez cerrado el arco. Se trata por tanto de ajustar la ley de presiones del arco para que coincida con la directriz proyectada en estado de cargas permanentes a tiempo infinito, esto es, una vez desarrollados los fenómenos reológicos del hormigón, eliminando por tanto los momentos flectores en el arco [4].

En este caso, se optó por prescindir de la realización de la apertura en clave del arco, operación compleja en este caso por la magnitud de las dimensiones del arco y los esfuerzos axiles asociados. En su lugar, la directriz del arco se calculó teniendo en cuenta el proceso constructivo y las fuerzas introducidas por los tirantes, de tal forma que la línea de presiones una vez acabada la construcción del arco fuese la deseada. Hay que recalcar que en este caso, no se buscaba que la ley de flectores bajo carga permanente fuera completamente nula, esto es, que la directriz del arco fuese la antifunicular perfecta de las cargas permanentes. Debido a que la ley de flectores provocada por la sobrecarga ferroviaria no es simétrica, siendo mayores los momentos flectores positivos que negativos en los arranques del arco, resulta conveniente que la ley de momentos flectores de carga permanente sea negativa en los arranques del arco (figura 10).

\subsection{Vinculación longitudinal}

En la situación definitiva el tablero se une con el arco en la clave del mismo, lo que permite utilizar el arco como transmisor 


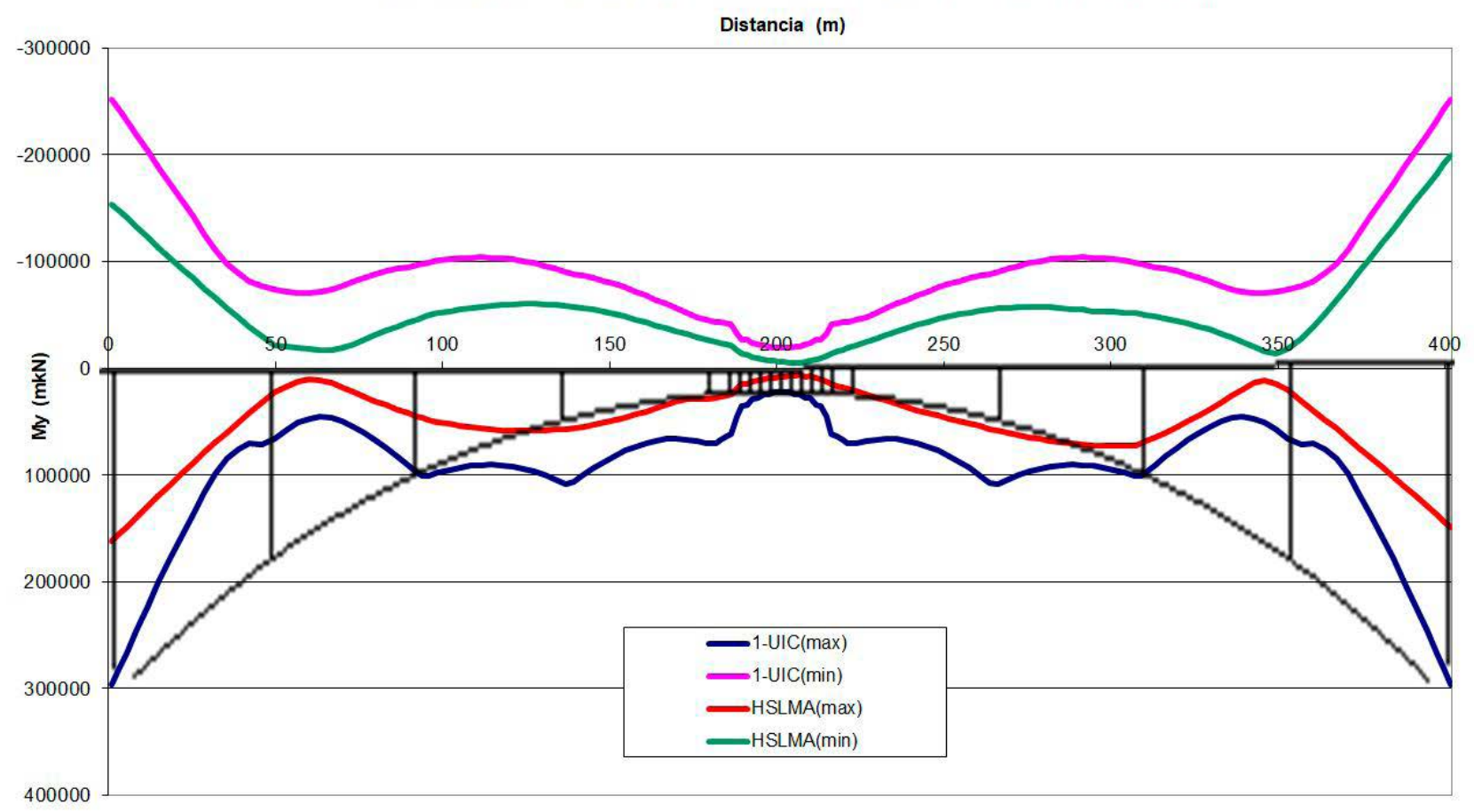

Figura 10. Esfuerzos en el arco debidos a la sobrecarga ferroviaria.

de las fuerzas horizontales de frenado y arranque, así como las provocadas por las fuerzas de rozamiento en los aparatos de apoyo, desde el tablero a la cimentación. Esta configuración es muy beneficiosa para la explotación y mantenimiento de la vía, porque implica que, siendo el viaducto de prácticamente $1 \mathrm{~km}$ de longitud, dispone de aparatos de dilatación de vía únicamente en estribos, siendo estos convencionales debido a la limitada distancia entre el punto fijo y los aparatos de dilatación de vía ( $453 \mathrm{~m}$ en el caso del estribo 1 y $543 \mathrm{~m}$ en el del estribo 2). Los análisis de interacción vía estructura llevados a cabo permitieron validar esta disposición comprobándose que las tensiones en el carril son admisibles.

Sin embargo, esta configuración no es posible mantenerla durante el proceso constructivo, y es necesario disponer puntos fijos provisionales. En una primera situación el punto fijo se sitúa en cada uno de los estribos. Cuando el tablero alcanza las pilas 6 y 15 (adyacentes al arco), el punto fijo se traslada a estas pilas, por ser el lugar donde se van a situar las torres de atirantamiento provisionales, liberando las coacciones longitudinales en estribos. Por último, cuando se materializa la unión arco-tablero, se liberan las coacciones en las pilas, llegando a la configuración definitiva (figura 11).

Esta secuencia constructiva obliga a diseñar tanto en estribos como en las pilas 6 y 15 unos mecanismos que permitan fijar el tablero longitudinalmente, y que puedan ser rápidamente liberados cuando se materialice el siguiente punto fijo, para garantizar que la estructura no dispone en ninguna situación de dos puntos fijos que impliquen sobreesfuerzos en la subestructura debido a los efectos térmicos.

El cambio en posición de los puntos fijos de la estructura requiere también un análisis detallado de los movimientos estimados teniendo en cuenta tanto las diferentes posiciones del punto fijo durante el proceso constructivo como las variacio- nes térmicas que pudieran producirse y que pueden llegar a ser acumulativos por la secuencia de puntos fijos, debiendo considerarse todas las hipótesis extremas para no infradimensionar las bandejas de los aparatos de apoyo (figura 11).

\subsection{Comportamiento de la estructura frente a las cargas de viento}

En estructuras de la magnitud de la que nos ocupa, el comportamiento frente a las cargas de viento es un factor clave en el diseño. El canto del arco debe ser lo más reducido posible para presentar una menor superficie expuesta al viento, y la forma de la sección transversal debe optimizarse en base al conocimiento de las propiedades del flujo del viento con un perfilado efectivo como para establecer un arco compacto y opaco al viento.

Con el fin de optimizar el comportamiento de la estructura ante las cargas de viento, en la fase de proyecto del arco del viaducto de Almonte se consideró como parámetro fundamental de diseño el reducir en todo lo posible el canto del arco, dotando además a la sección transversal de unos biseles que redujesen su coeficiente de arrastre. La sección debe provocar la mínima perturbación a la circulación del aire, reduciendo su canto y optimizando su forma, pero además debe poseer la inercia suficiente para hacer frente a los momentos flectores concomitantes provenientes de las cargas de tráfico, según se ha expuesto anteriormente.

Todos estos aspectos se estudiaron durante la fase de proyecto, mediante la realización de ensayos seccionales y globales sobre modelo reducido.

Descartada la sección maciza para el arco, por criterios estructurales de peso y eficiencia estructural debido al canto necesario, el diseño de la sección buscó cumplir los requisitos 

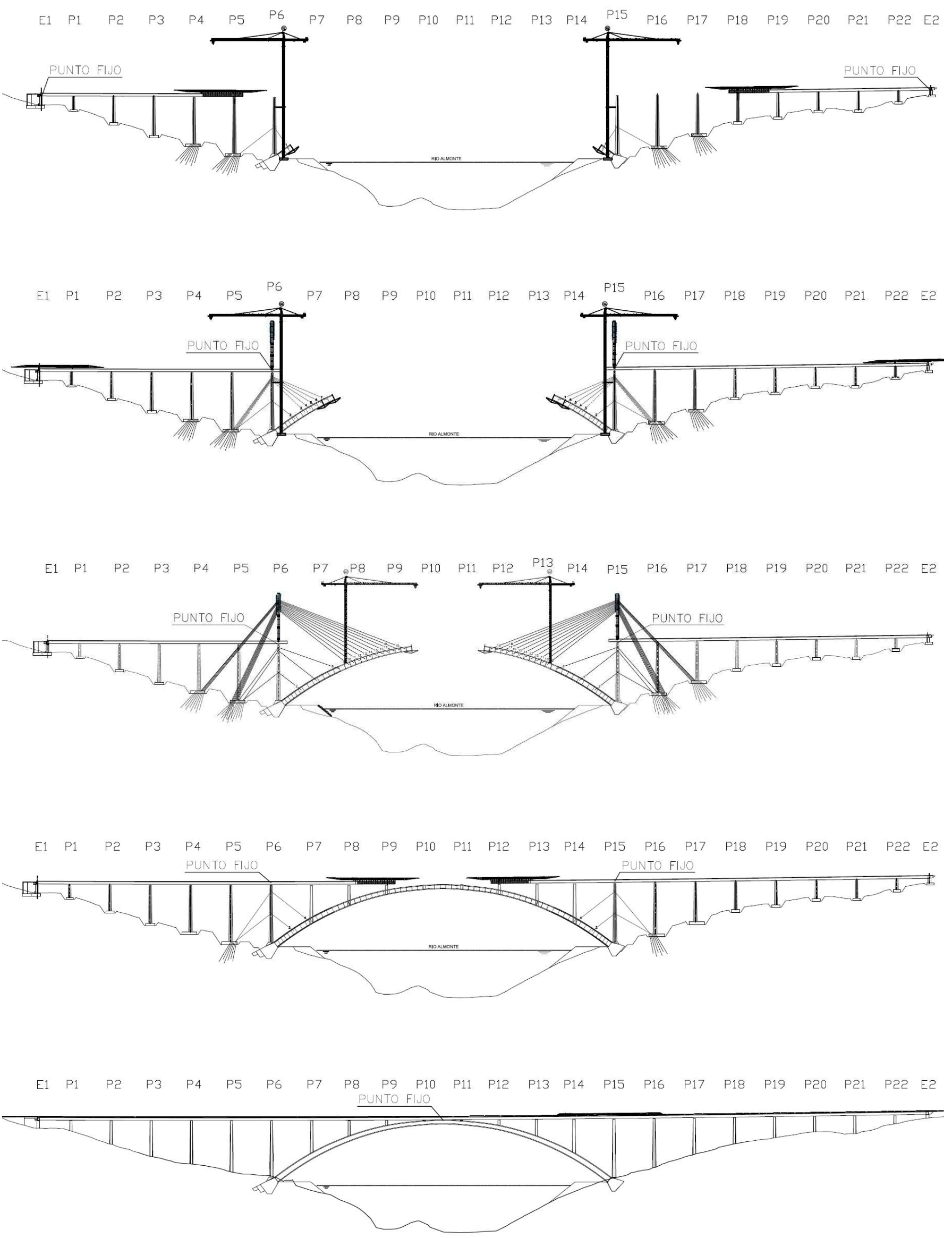

Figura 11. Evolución del punto fijo longitudinal de la estructura. 


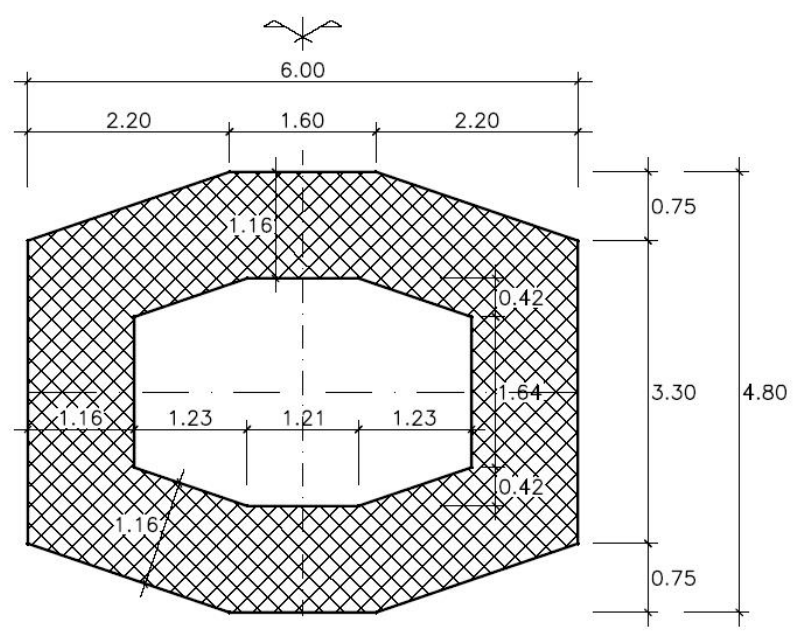

Figura 12. Sección en clave del arco. Relación entre canto y chaflanes.
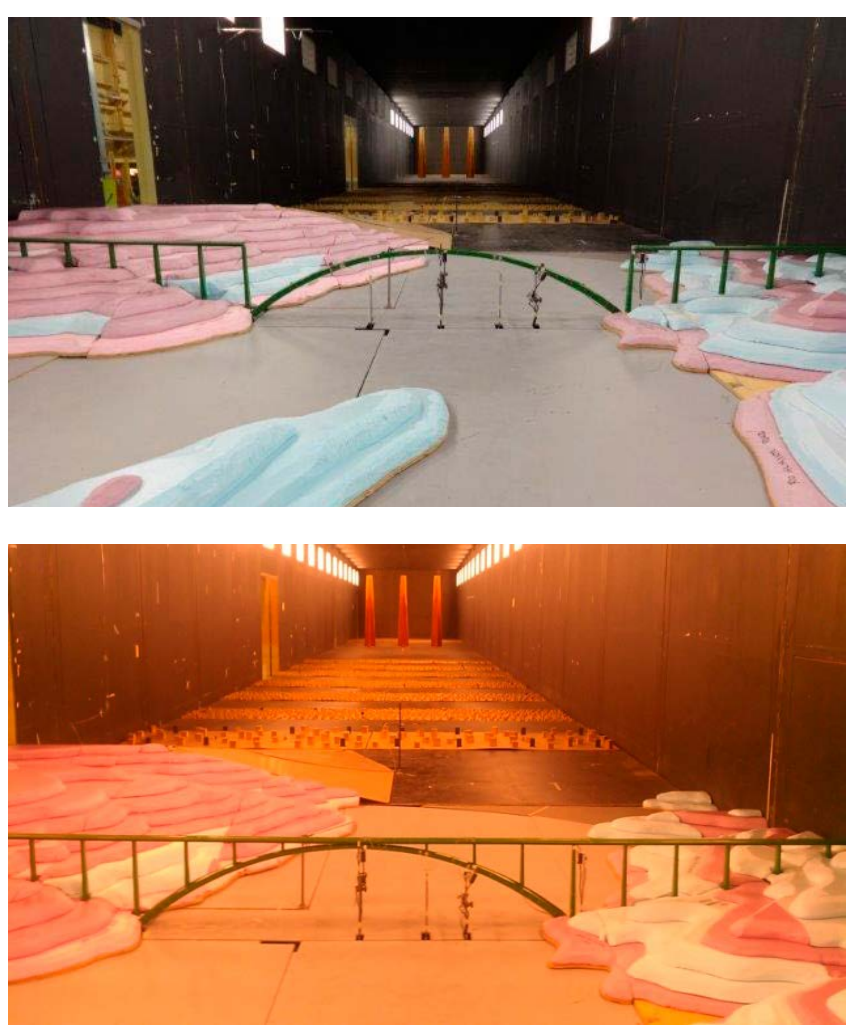

Figura 13. Túnel de viento en situación constructiva y definitiva.

de máxima compacidad y, por tanto, perturbación menor del flujo aéreo.

El resultado de todo el análisis anterior conduce a que el puente sobre el río Almonte tenga una sección hueca de forma alargada y con chaflanes pronunciados que afilan su perfil para los vientos de dirección horizontal (figura 12).

El estudio de los efectos del viento en el viaducto se llevó a cabo mediante la realización de ensayos de modelo seccional y de un modelo aeroelástico integral, que recogía tanto las fases de construcción como la del puente en su configuración final. Dada la topografía en la ubicación del puente, y su efecto en las condiciones del viento, el modelo incluía también el terreno en la zona de estudio. El modelo integral permitió el análisis de la respuesta dinámica del puente completo conforme a la turbulencia atmosférica en tres dimensiones (Figura 13).

\section{4. \\ ANÁLISIS ESTRUCTURAL}

\subsection{Cálculo no lineal en ELU y ELS}

En elementos cuya respuesta estructural está significativamente influenciada por los efectos de $2^{\circ}$ orden, como son los arcos por ser estructuras que trabajan por forma, las condiciones de equilibrio y compatibilidad deben ser comprobadas sobre la estructura deformada, tanto para las situaciones de servicio como para los estados límite últimos.

El cálculo no lineal realizado tiene en cuenta tanto la no linealidad geométrica (originada por el cambio de la geometría debido a las deformaciones), como la no linealidad de las propiedades del material en ELU, incluyendo los efectos de la fisuración en el hormigón y la no linealidad de las curvas tensión-deformación, tanto en arco como en tablero. La no linealidad del comportamiento del hormigón (debido a la fisuración del hormigón, no linealidad a compresión y plastificación de las armaduras) obliga a realizar a un proceso incremental de carga, en el que se asegura el equilibrio y la compatibilidad de deformaciones en todas las fases del proceso, recorriendo los rangos elástico, fisurado y previo al agotamiento.

Por tratarse de una estructura muy compleja en la que cobran especial importancia los efectos no lineales, y donde no resulta aplicable el método simplificado propuesto en la normativa española (EHE) para el cálculo del estado límite de inestabilidad, es necesario abordar el análisis de $2^{\circ}$ orden mediante el método general (no desarrollado en la normativa española), con la consideración tanto de la no linealidad geométrica como de la no linealidad del material, y que implica un tratamiento de seguridad diferente al propuesto en la Instrucción EHE, como se indica en el artículo 19.2.2

Por ello, para realizar los cálculos en estado límite último es necesario recurrir al método general desarrollado en las versiones EN (euronormas) de los Eurocódigos, en concreto del Eurocódigo 2 para estructuras de hormigón en sus partes EN1992-1 (reglas generales y reglas para edificación) y EN1992-2 (puentes).

El modelo de cálculo tridimensional realizado (figuras 14 y 15) considera todos los efectos determinantes:

- La fisuración del hormigón, evaluando la rigidez de cada sección del arco y tablero en cada una de las fases de carga, atendiendo a su fisuración y adaptando su inercia.

- La retracción y fluencia del hormigón.

- La no linealidad de las ecuaciones constitutivas $(\sigma-\varepsilon)$ del acero y del hormigón (ver apartado 4.1.6).

- Los efectos de las fases y secuencias de montaje en la seguridad final de la estructura terminada.

\subsubsection{Análisis de la seguridad del arco en estado límite últi- mo (Formato EN 1992-1-1)}

El EN 1992-1-1 establece que los análisis no lineales de $2^{\circ}$ orden se realicen con modelos que reproduzcan de forma "realista" la rigidez de las secciones transversales de la estructura, 


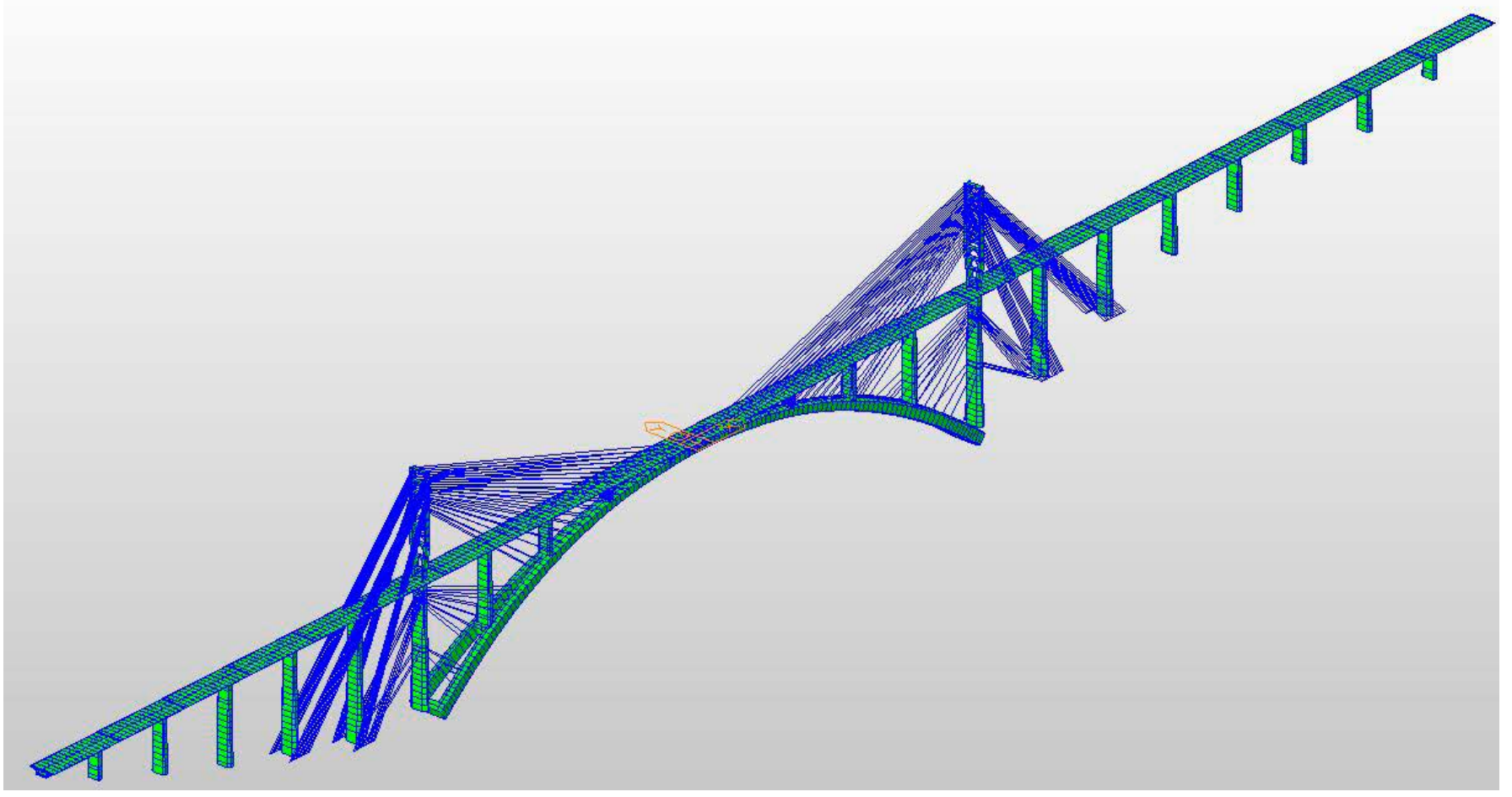

Figura 14. Vista general del modelo de cálculo tridimensional.

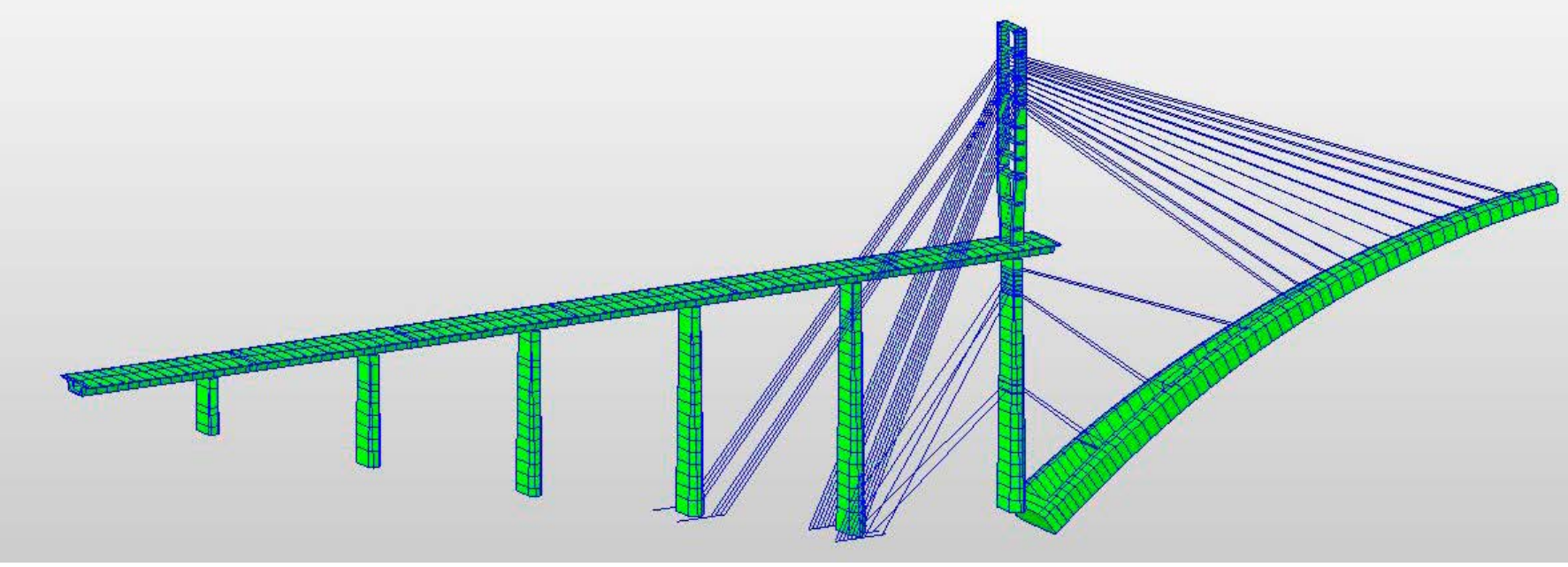

Figura 15. Vista de detalle de una fase intermedia del proceso constructivo.

tanto en la fase elástica inicial como en las etapas posteriores no elásticas, considerando las resistencias medias del hormigón y acero. Posteriormente, para la comprobación resistente de las secciones frente a los esfuerzos solicitantes, se establece la necesidad de utilizar la resistencia minorada de los materiales para tener la seguridad necesaria en la estructura frente a fallos localizados de la calidad de los materiales.

Para ello se realizaron modelos no lineales con un proceso incremental de carga, en el que se asegura el equilibrio y la compatibilidad de deformaciones en todas las fases del proceso. Cada uno de los distintos modelos tenía como objeto introducir la combinación de acciones pésima para una determinada sección del arco, con la imperfección geométrica en el arco que maximizase igualmente los esfuerzos en la sección de estudio.
En línea con lo reflejado en el EN1992-1-1 y en el Código Modelo del 90, se procedió a realizar los modelos de análisis en $2^{\circ}$ orden con resistencias y rigidices medias y, posteriormente, comprobar la resistencia de las secciones con resistencias minoradas de los materiales. Las etapas de cálculo consistieron en: 1. Análisis no lineal de todas las fases constructivas hasta llegar al estado final de cargas permanentes incorporando las imperfecciones geométricas iniciales.

2. Análisis diferido no lineal, geométrico y del material, de las redistribuciones por fluencia y retracción del estado de solicitaciones del apartado anterior.

3. Análisis no lineal, geométrico y del material, de las acciones térmicas.

4. Análisis no lineal general, paso a paso, a partir de la geometría y del estado de presolicitaciones obtenidas al 


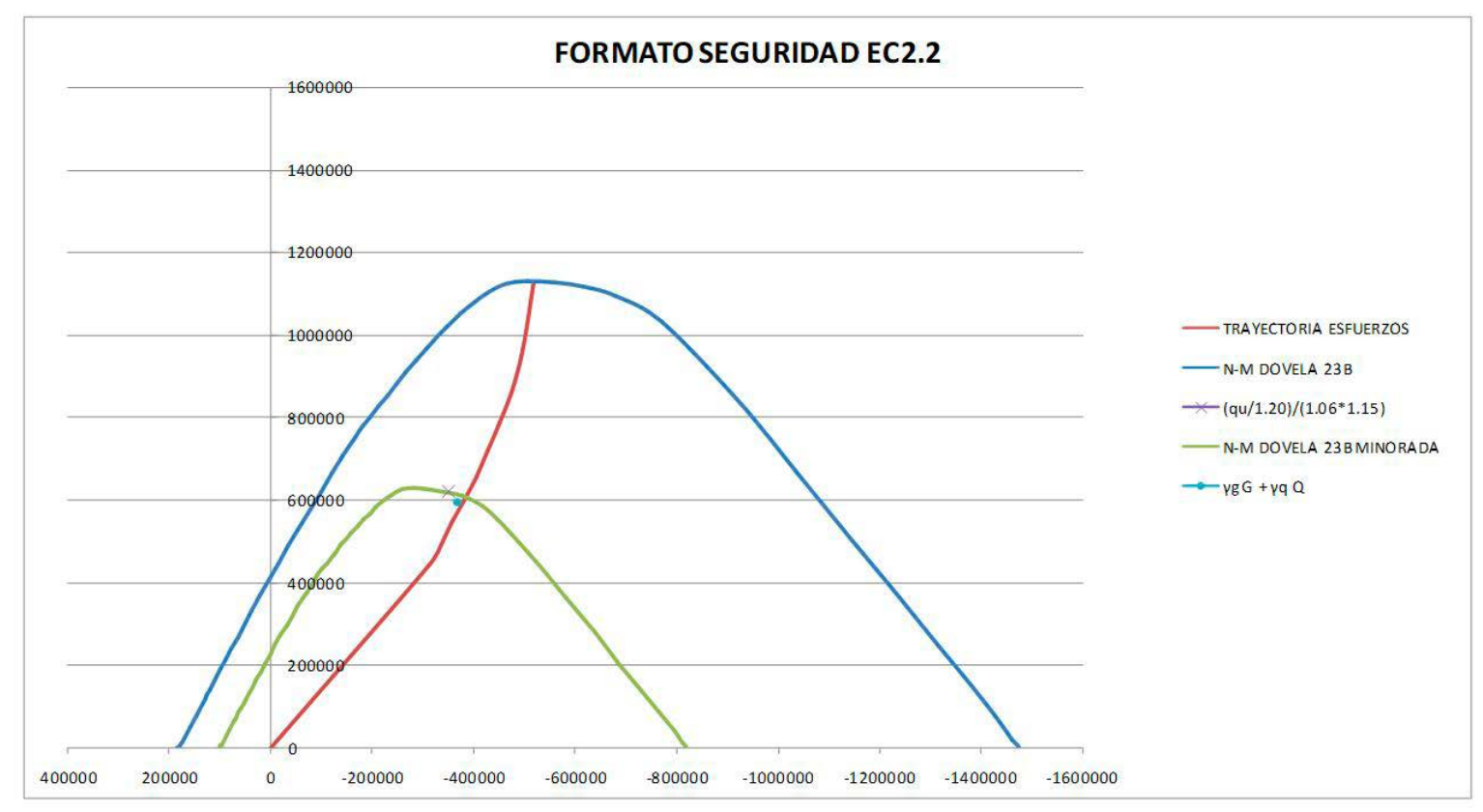

Figura 16. Coeficiente de seguridad de la sección bajo P12 según el formato de seguridad EN1992-2.

final del apartado anterior. Para ello se introducen las cargas correspondientes a las cargas permanentes desde sus valores característicos hasta sus valores mayorados, y a las sobrecargas de uso, mediante incrementos escalonados, a partir de los valores del apartado 3 , de forma que se alcanzan los valores de ELU en el mismo paso. En cada etapa de carga se realiza una adaptación de la rigidez de cada una de las secciones del modelo a partir de las leyes tensión-deformación propuestas por el Eurocódigo 2 , tanto para el acero como para el hormigón, donde se considera la fisuración del hormigón en las secciones donde exista.

5. Finalmente se comprueban las secciones bajo los esfuerzos totales debido a las acciones mayoradas, adoptando modelos convencionales de cálculo de la resistencia de las secciones con resistencias minoradas de los materiales.

\subsubsection{Análisis de la seguridad global (Formato EN 1992-2)}

Como comprobación adicional de la estructura, en la fase de construcción y conjuntamente con los Servicios Técnicos de FCC, se procedió a realizar el análisis con los nuevos formatos de seguridad para análisis no lineal de estructuras de hormigón recogidos en el Eurocódigo 2 parte 2, y el Código Modelo 2010. Dicho formato usa características medias para el acero y un valor de resistencia media minorada para el hormigón de $0.84 \mathrm{f}_{\mathrm{ck}}$, con el objeto de "homogeneizar" su coeficiente de seguridad parcial al del acero, y así obtener un coeficiente de seguridad global de la estructura, incrementando la carga hasta que la estructura alcance su resistencias última o hasta que se produzca un fallo global de la estructura.

Con el formato de seguridad EN 1992-2, se aumenta la carga por encima de las cargas de estado límite último hasta alcanzar el colapso de la estructura (para una carga $\mathrm{q}_{\mathrm{ud}}$ ), ya sea por alcanzar una sección de la estructura su resistencia última o porque se produzca un fallo global de la estructura por inestabilidad (pandeo). Debiendo cumplirse una de las siguientes condiciones, recogidas en el apartado 5.7 del EN 1992-2:2005 [5]:

$\gamma_{\text {Rd }} E\left(\gamma_{G} G+\gamma_{Q} Q\right) \leq R\left(\frac{q_{u d}}{\gamma_{0}}\right)$

(criterio A)

$E\left(\gamma_{G} G+\gamma_{Q} Q\right) \leq R\left(\frac{q_{u d}}{\gamma_{R d} \gamma_{0}}\right)$

(criterio B)

$\gamma_{\text {Rd }} \gamma_{S d} E\left(\gamma_{G} G+\gamma_{Q} Q\right) \leq R\left(\frac{q_{u d}}{\gamma_{0}}\right)$

(criterio C)

La seguridad de la estructura queda verificada con el cumplimiento de cualquiera de las tres condiciones $\mathrm{A} / \mathrm{B} / \mathrm{C}$ indicadas, porque el cumplimiento de todas las inecuaciones conduciría a estructuras sobredimensionadas (figura 16).

\subsubsection{Hipótesis de cálculo}

La no linealidad en el comportamiento real del puente obliga a descartar de inicio la posibilidad de recurrir a la clásica superposición de estados, por lo que se hace necesario establecer previamente las hipótesis de cálculo a analizar y sus combinaciones. En este caso, y atendiendo a los esfuerzos de primer orden, se definió en el arco un número amplio, aunque limitado, de secciones criticas y para cada una de ellas se determina la combinación de cargas para las que se alcanza tanto el momento flector máximo como el mínimo (figura 17a).

En las combinaciones consideradas se han tenido en cuenta las cargas habituales recogidas en la IAPF:

- Peso propio y cargas permanentes.

- Acciones reológicas (retracción y fluencia).

- Fuerzas procedentes del pretensado del tablero.

- Fuerza de rozamiento en apoyos.

- Temperatura y gradiente térmico en arco, pilas, pilastras y tablero. 


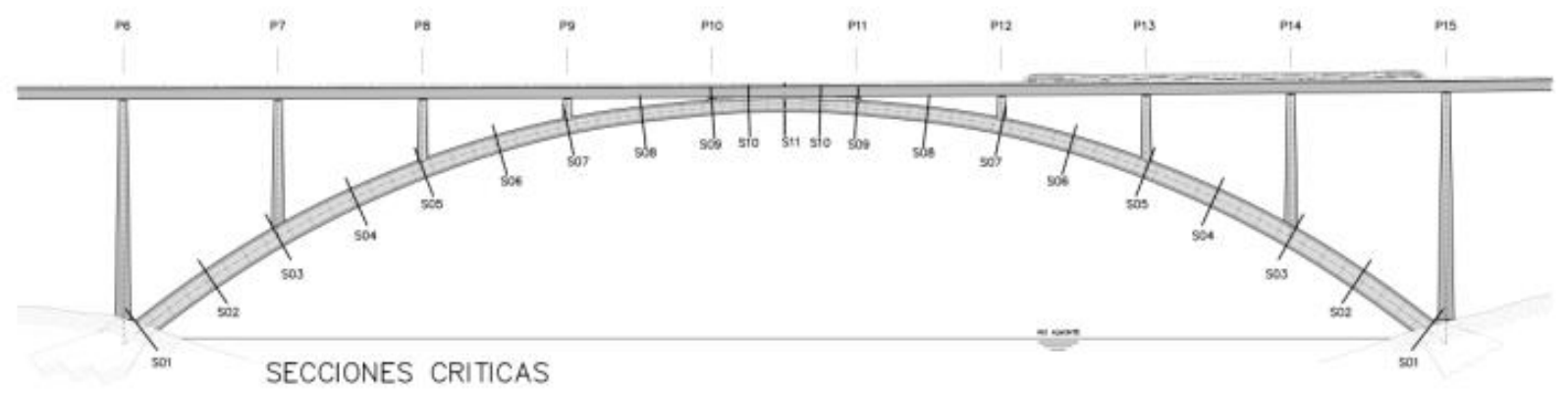

Figura 17a. Secciones críticas a estudiar.

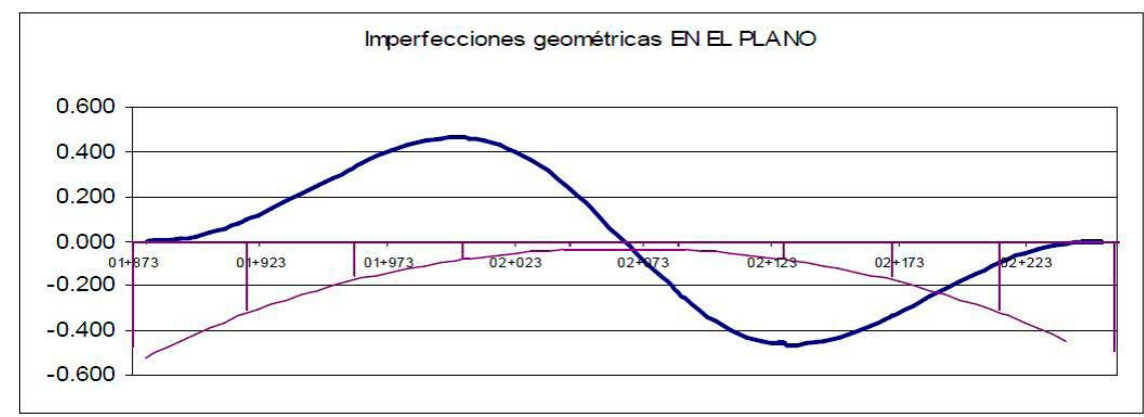

Figura 17b. Imperfección en el plano del arco.

- Sobrecargas de uso (2 trenes UIC-71, efecto lazo, frenado y arranque) .

- Sobrecarga en paseos de servicio.

- Viento longitudinal y transversal.

\subsubsection{Introducción de la no-linealidad geométrica. Imperfec- ción inicial}

Los análisis de $2^{\circ}$ orden deben realizarse teniendo en cuenta los efectos desfavorables de posibles desviaciones en la geometría de la estructura.

A este respecto, el apartado 5.2 del Eurocódigo 2 establece que los análisis de $2^{\circ}$ orden deberán tener en cuenta los efectos desfavorables de posibles desviaciones en la geometría de la estructura, o en la posición (excentricidad) de la línea de acción de las cargas (línea de presiones), en las verificaciones de la seguridad de la pieza en estados límite últimos.

La EHE 2008 no indica nada en cuanto a imperfecciones iniciales en arcos de hormigón, por lo que no resulta aplicable.

Para estructuras de hormigón, dicha imperfección viene recogida en el apartado 5.2 de la parte 2 (Puentes) del Eurocódigo 2 (EN1992-2), y propone para el caso de puentes arco la idealización de la imperfección geométrica inicial mediante una función sinusoidal basada en la forma del primer modo de pandeo, con una amplitud equivalente a una excentricidad seccional elástica de valor $\mathrm{a}_{0}$ (figura 17b).

$\mathrm{a}_{0}=\theta_{0} \alpha_{\mathrm{h}} \frac{\mathrm{L}_{\mathrm{p}}}{2}=\frac{1}{200} 0.183 \frac{120}{2}=0.055 \mathrm{~m} \quad$, donde

$\theta_{0}=1 / 200$ (valor base)

$\mathrm{L}_{\mathrm{p}}=$ semilongitud efectiva de la onda del modo de pandeo $(120 \mathrm{~m})$

$\alpha_{h}=$ coeficiente reductor en función de la longitud de la estructura $\alpha_{\mathrm{h}}=2 / \sqrt{ } \mathrm{L}_{\mathrm{p}}=0.183$ $\mathrm{a}_{0}^{*}=\mathrm{a}_{0} \frac{\mathrm{N}_{\mathrm{cr}}}{\mathrm{EI} \eta_{\mathrm{cr}}^{\prime \prime}} \eta_{\mathrm{cr}}=0.055 \cdot 1.695=0.093 \mathrm{~m} \quad$, donde

$\mathrm{a}_{0}^{*} \quad$ es la amplitud de la imperfección geométrica equivalente, a imponer a la directriz del arco para conseguir la imperfección $\mathrm{a}_{0}$ sobre la línea de presiones

El procedimiento de cálculo seguido consiste en introducir la imperfección geométrica equivalente del arco en la directriz del modelo de cálculo evolutivo para, de esta forma, realizar el cálculo fase a fase con la geometría predeformada, no existiendo por tanto un estado de cargas artificial que intente reproducir los efectos de la excentricidad.

La imperfección inicial a considerar es aquella que mayor amplificación de esfuerzos produzca en la sección de estudio, y por tanto es distinta para cada una de las hipótesis de carga estudiadas, siendo siempre la predeformada considerada homotética al primer modo de pandeo de cada una de las hipótesis de carga. A continuación, se muestra el modo de pandeo considerado para una de las hipótesis analizadas, siendo la predeformada para ese caso de carga una réplica ponderada de esa deformada (figura 18).

En cuanto a la imperfección transversal del arco (fuera del plano), los análisis realizados determinaron que no resultaba significativa para el dimensionamiento y comprobación de la estructura. Esto es debido fundamentalmente a que para mejorar la respuesta de la estructura frente a fenómenos de inestabilidad fuera del plano, el arco se diseñó con una bifurcación transversal de su sección en los arranques, lo que aumenta de manera muy importante la rigidez transversal del mismo.

\subsubsection{Análisis de la sensibilidad de la estructura frente a las imperfecciones iniciales}

A pesar de que el valor de la imperfección geométrica a utilizar en el cálculo del viaducto viene claramente recogido en 


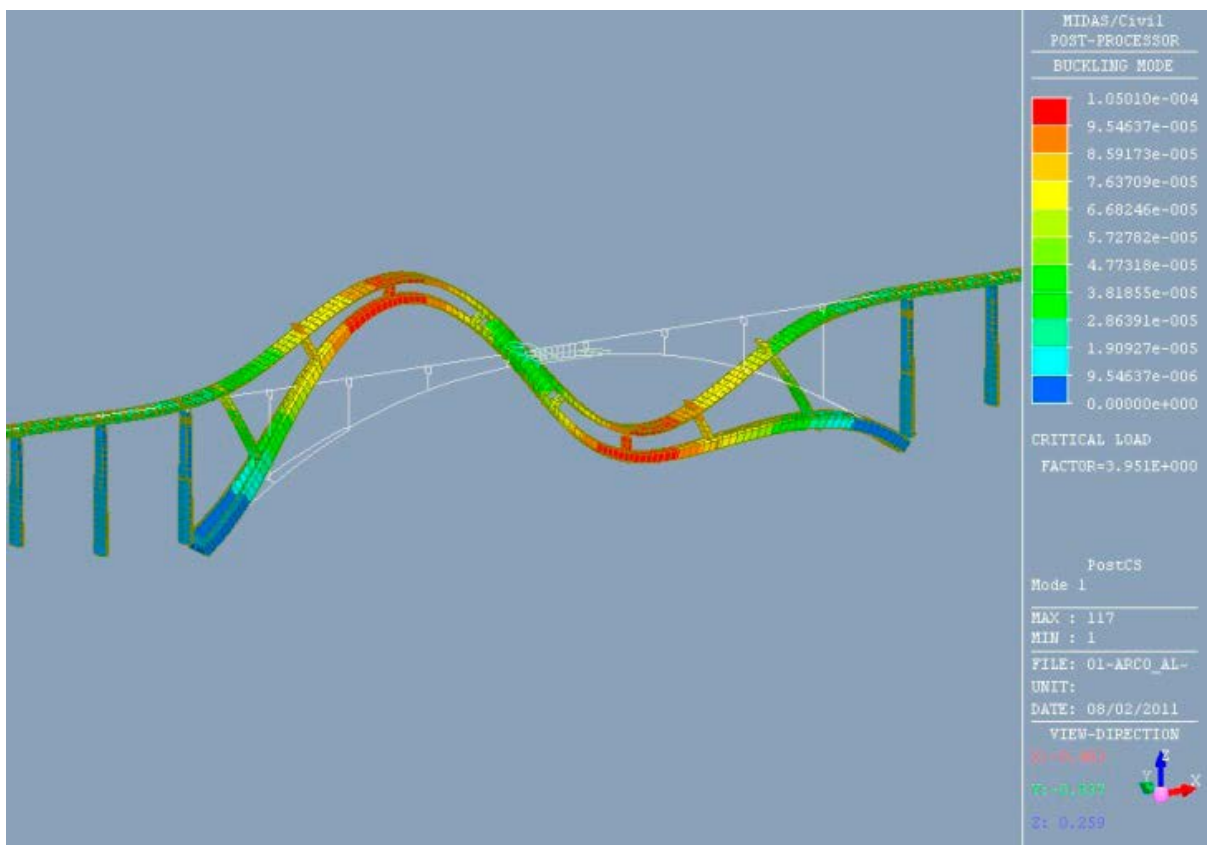

Figura 18. Modo de pandeo correspondiente a la hipótesis denominada S08-Min.

el apartado 5.2 de la parte 2 (Puentes) del Eurocódigo 2, dedicado al cálculo y disposiciones constructivas de puentes de hormigón, y es como se indica en el punto anterior de $9.3 \mathrm{~cm}$, es un parámetro de cálculo de una importancia fundamental en el comportamiento de la estructura y por ello fue objeto de un estudio detallado.

Los valores de imperfección recogidos en la Normativa dependen de la luz del arco, pero son independientes del proceso constructivo elegido para la ejecución del mismo, siendo por tanto iguales para un puente ejecutado con cimbra, con pilas provisionales, por avance en voladizo o con torre de atirantamiento. Por tanto, los valores recogidos en la Normativa deben ser aplicados en casos de obras tan singulares como ésta con la lógica prudencia. Tampoco las experiencias anteriores en otros arcos de puentes carreteros de gran luz son plenamente aplicables a este viaducto, por las particularidades intrínsecas de este proyecto al tratarse de un puente ferroviario con unas sobrecargas móviles muy elevadas que producen ya una variación importante de la línea de presiones adicional a las imperfecciones consideradas.

Por otra parte, y dada la magnitud del arco del viaducto del Almonte y el sistema constructivo con torres de atirantamiento y grandes voladizos atirantados, los movimientos cíclicos diarios por efectos térmicos son muy importantes, así como los movimientos posteriores a la ejecución de la última dovela por efectos reológicos. Si bien todos estos movimientos son simétricos y por tanto un error geométrico por mala compensación de estos efectos no excitan el primer modo de pandeo, se consideró necesario realizar un estudio detallado de la sensibilidad de la estructura frente a un incremento de la imperfección normativa ante la posibilidad de que los errores geométricos superasen la imperfección asimétrica de amplitud $9.3 \mathrm{~cm}$.

Resulta interesante destacar que el Eurocódigo 2 en la parte general (EN1992-1-1), anterior al EN1992-2, establece la misma formulación para el cálculo de la imperfección inicial de cálculo, pero con el matiz de establecer para el coeficiente reductor en función de la longitud de la estructura $\left(\alpha_{\mathrm{h}}\right)$ un valor inferior de $2 / 3$. Esto da lugar a un valor bastante superior de la imperfección geométrica:

$$
\begin{aligned}
& \mathrm{a}_{0}=\theta_{0} \alpha_{\mathrm{h}} \frac{\mathrm{L}_{\mathrm{p}}}{2}=\frac{1}{200} \frac{2}{3} \frac{120}{2}=0.200 \mathrm{~m} \\
& \theta_{0}=1 / 200 \\
& \mathrm{~L}_{\mathrm{p}}=120 \mathrm{~m} \\
& \alpha_{\mathrm{h}}=2 / \sqrt{\mathrm{L}_{\mathrm{p}}}=0.183, \text { cumpliendo } 2 / 3 \leq \alpha_{\mathrm{h}} \leq 1 \\
& \mathrm{a}_{0}^{*}=\mathrm{a}_{0} \frac{\mathrm{N}_{\mathrm{cr}}}{\mathrm{EI}_{\mathrm{cr}}^{\prime \prime \prime}} \eta_{\mathrm{cr}}=0.200 \cdot 1.695=0.339 \mathrm{~m}
\end{aligned}
$$$$
\text { , donde }
$$

Según se recoge en el "Designers' guide to EN1992-2" [6], esta limitación en el coeficiente $\alpha_{h}$ se eliminó en el EN1992-2, para evitar las excesivas imperfecciones a las que daba lugar en elementos de gran longitud.

Por todo lo anteriormente explicado, y para garantizar una seguridad suficiente de la estructura ante posibles errores geométricos de construcción que no pudiesen ser corregidos durante las diferentes fases constructivas, se decidió calcular la estructura con una imperfección geométrica de $33.9 \mathrm{~cm}$, realizando incluso un estudio de sensibilidad de la estructura utilizando una imperfección un $50 \%$ mayor $\left(\mathrm{a}_{0}^{*}=1.5 \times 0,339=0.510 \mathrm{~m}\right)$, para asegurar que la estructura no sufriese un descenso brusco de la seguridad al pandeo ante el incremento de la imperfección.

Finalizada la ejecución de la estructura, se ha podido comprobar que el error de ejecución asimétrico medido es de $8 \mathrm{~cm}$, pudiendo concluirse que las hipótesis de diseño han resultado conservadoras.

\subsubsection{Introducción de la no-linealidad del material en los modelos de estado límite último}

La respuesta no lineal de los materiales se considera mediante la adopción de leyes de comportamiento elastoplásticas para la 


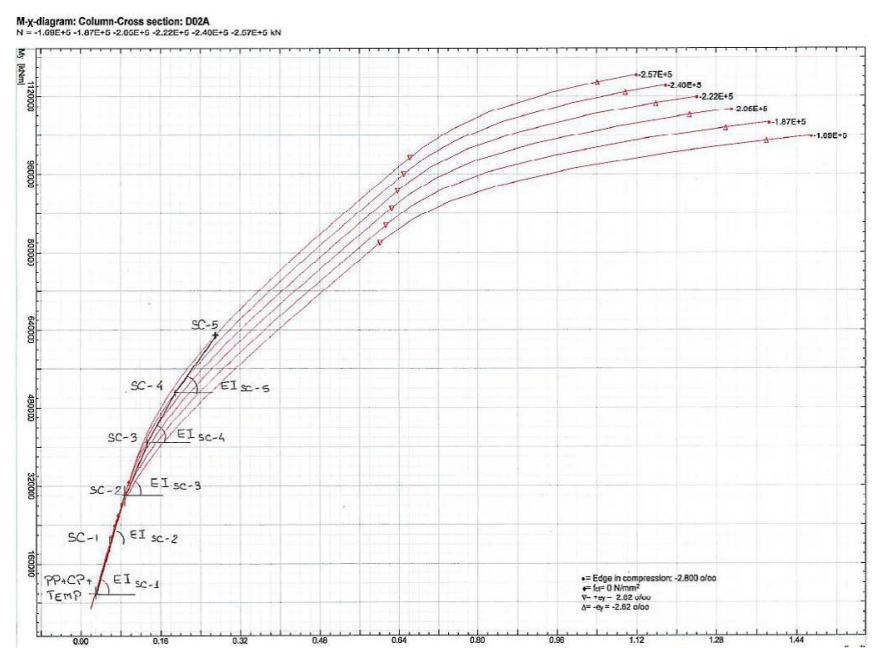

Figura 19. Diagrama Momento-Curvatura para la Dovela 02.

obtención de las leyes axiles-momentos-curvatura de las secciones transversales del arco y el tablero.

Como se ha explicado anteriormente, la no linealidad del comportamiento del hormigón en ELU (debido a la fisuración del hormigón, no linealidad a compresión y plastificación de las armaduras) obliga a proceder a un proceso incremental de carga. En este análisis incremental, las acciones correspondientes a las cargas permanentes deben aplicarse desde sus valores característicos hasta sus valores mayorados, y se deben introducir simultáneamente las sobrecargas de uso mediante incrementos escalonados de forma que se alcanzan los valores de ELU en el mismo paso. En cada etapa de carga se realiza una adaptación de la rigidez de cada una de las secciones del modelo a partir de las leyes tensión-deformación propuestas por el Eurocódigo 2 tanto para el acero como para el hormigón, donde se considera la fisuración del hormigón.

Se trata, por tanto, de un proceso incremental-iterativo, ya que inicialmente no existe fisuración, y se comprueba, con los esfuerzos obtenidos para cada fase y los diagramas momento-curvatura de cada sección, si esta hipótesis es correcta. Se corrigen los valores de la rigidez de la fase correspondiente, adaptándose al diagrama tensión-deformación del EC-2, y se procede a calcular de nuevo los esfuerzos, comprobando si las rigideces consideradas corresponden con los niveles tensionales alcanzados. Este proceso se repite hasta que la variación de rigidez en cada fase entre el valor considerado para el cálculo de esfuerzos y el que se obtendría del diagrama momento-curvatura es despreciable.

En este procedimiento incremental de cálculo se toman las rigideces tangentes a la gráfica momento-curvatura en el punto correspondiente al incremento de carga anterior, tal y como se muestra en las siguientes figuras, donde se representan los diagramas momento-curvatura para los diferentes niveles de axil en la Dovela 2 (fisurada en el incremento de carga SC-3) y la Dovela 7 (no fisurada en todo el proceso para esta hipótesis de cálculo) en cada uno de los incrementos de carga. Se indican los momentos flectores solicitantes, así como las rigideces consideradas en el cálculo de los mismos en la última iteración para la hipótesis de cálculo considerada (figuras 19 y 20).

Tanto en el modelo de cálculo como en la determinación de diagramas momento-curvatura se empleó un diagrama tensión-deformación del hormigón adaptado al cálculo no lineal, basado en las resistencias medias del hormigón y el acero, tal

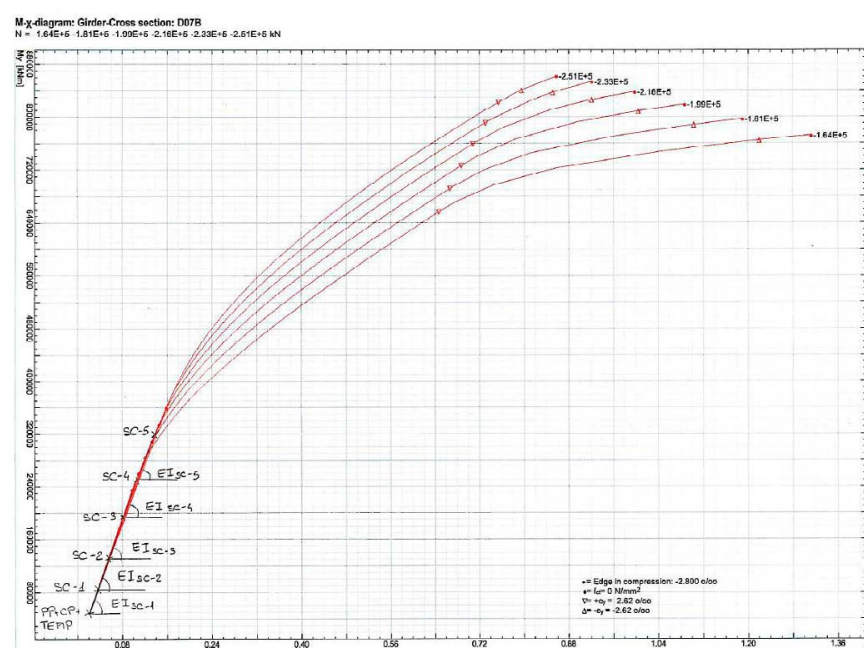

Figura 20. Diagrama Momento-Curvatura para la Dovela 07.

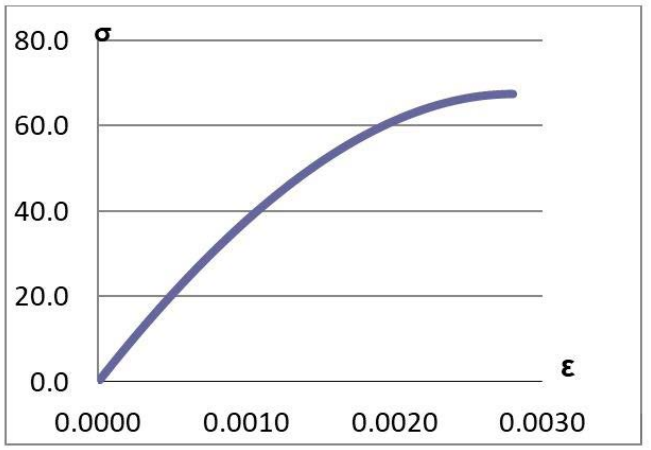

Figura 21a. Diagramas $\sigma-\varepsilon$ del hormigón HA-80 para el cálculo no lineal.

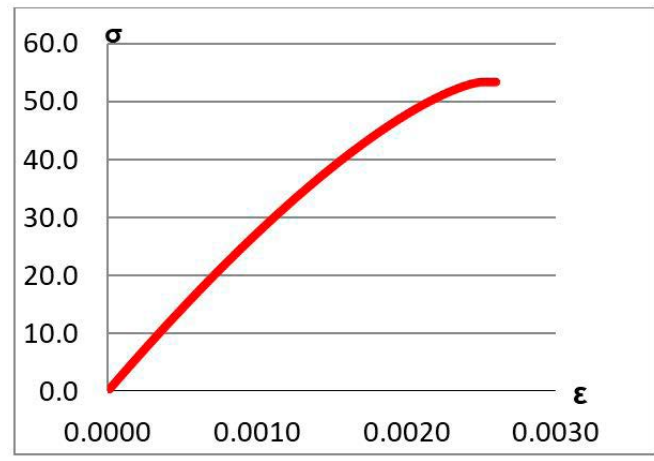

Figura 21b. Diagramas $\sigma-\varepsilon$ del hormigón HA-80 para el cálculo seccional.

y como se indica en el EN 1992-2 Apartado 5.7 [5] y se ha explicado anteriormente (figura 21a).

Para el cálculo seccional se empleó el diagrama parábola-rectángulo, con resistencias minoradas de los materiales (figura 21b).

\subsection{Consideración del proceso constructivo}

La importancia del proceso constructivo en el comportamiento de la estructura obliga a incorporar en el análisis no lineal anteriormente descrito todas las fases constructivas, generando la geometría del arco durante el proceso constructivo con las imperfecciones geométricas indicadas por la normativa. 
Adicionalmente, fue necesario desarrollar un modelo de cálculo del proceso constructivo con todas las subfases de hormigonado de dovela modelizadas. La necesidad de un detallado análisis del proceso constructivo no radica únicamente en la verificación resistente de la estructura durante las fases constructivas, sino que es fundamental para el correcto control geométrico que garantice que la geometría final conseguida es la definida en proyecto, y que funcione como antifunicular de las cargas permanentes consiguiendo por tanto que todos los puntos de las distintas secciones del arco se encuentren en compresión.

Así, el modelo de cálculo detallado del proceso constructivo, desarrollado conjuntamente con los Servicios Técnicos de FCC, incorpora las cargas necesarias para modelizar con precisión todas las etapas que se producen en la ejecución de cada dovela del arco: colocación del carro, armado, movimiento del encofrado, y la colocación y tesado de los tirantes. Este modelo detallado es necesario para la determinación de las contraflechas en cada una de las fases, la correcta colocación del carro de avance así como las correcciones geométricas necesarias en cada una de las fases por los efectos térmicos.

El criterio adoptado para el diseño de las etapas del proceso constructivo es que en ningún caso las tracciones máximas en cualquier fibra del arco frente a la combinación frecuente de acciones, teniendo en cuenta las cargas permanentes y las acciones variables, resulten superiores a la resistencia característica media a tracción del hormigón $\left(\mathrm{f}_{\mathrm{ctm}}\right)$, de tal manera que se evite la fisuración del arco.

En cuanto a los tirantes, por ser estos provisionales, se decidió diseñarlos sin vaina común exterior, para reducir el coste y facilitar las operaciones de montaje y desmontaje. Se utilizaron cordones de acero de $150 \mathrm{~mm}^{2}$ de sección y resistencia de 1860 $\mathrm{MPa}$. El dimensionamiento de los mismos se hizo de tal manera que el valor de la tensión máxima para cada uno de los tirantes fuese inferior al 55\% de la tensión de rotura garantizada.

El procedimiento constructivo planteado en proyecto contemplaba la construcción del arco mediante avance en voladizo con torre de atirantamiento y 23 familias (tiro + retenida)

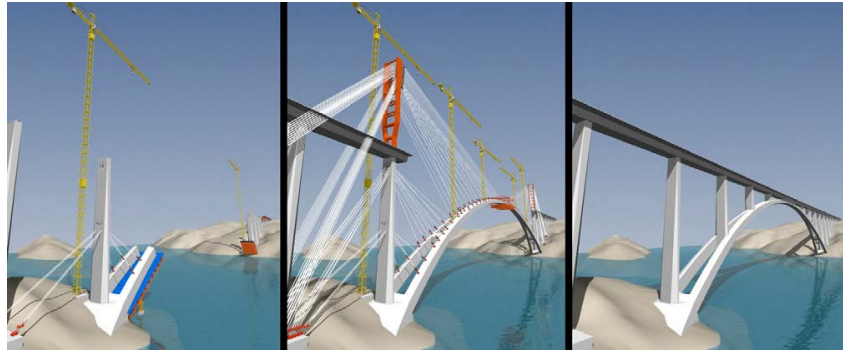

Figura 22. Infografías del procedimiento constructivo.

de tirantes provisionales. En la imagen se muestran las infografías de procedimiento constructivo desarrollado en la fase de proyecto. En fase de construcción se adaptó el procedimiento al tamaño de dovela de $6.40 \mathrm{~m}$ finalmente escogido, pasando de 23 a 26 familias de tirantes (figura 22).

La familia de tirantes y retenidas 25 y 26 se diseñaron para trabajar a una tensión inferior, con el objeto de poder actuar sobre ellos aumentando la fuerza de tesado previamente al cierre en clave, si fuese necesario realizar alguna corrección en la geometría del voladizo.

Para conseguir un correcto estado tensional de todas las dovelas durante las distintas fases del proceso constructivo, se comprobó que no era posible ejecutar el arco actuando únicamente en el tirante correspondiente al frente de fase en cada una de las etapas de avance del carro. Era por tanto necesario realizar ajustes en las fuerzas de los tirantes colocados en fases previas para evitar fisuraciones en zonas intermedias del arco ya construido. La optimización de estos procedimientos pasa por minimizar el número de operaciones. Por ello, se redujeron el número de retesados de tirantes al mínimo posible ( 2 familias) y todos los destesados que se realizaron fueron totales. En la fase de ejecución de la dovela de cierre, únicamente permanecían activas 17 de las 26 familias de tirantes que eran necesarias para la ejecución del arco, habiéndose ido retirando las 9 familias restantes en distintas fases del proceso constructivo (figura 23).
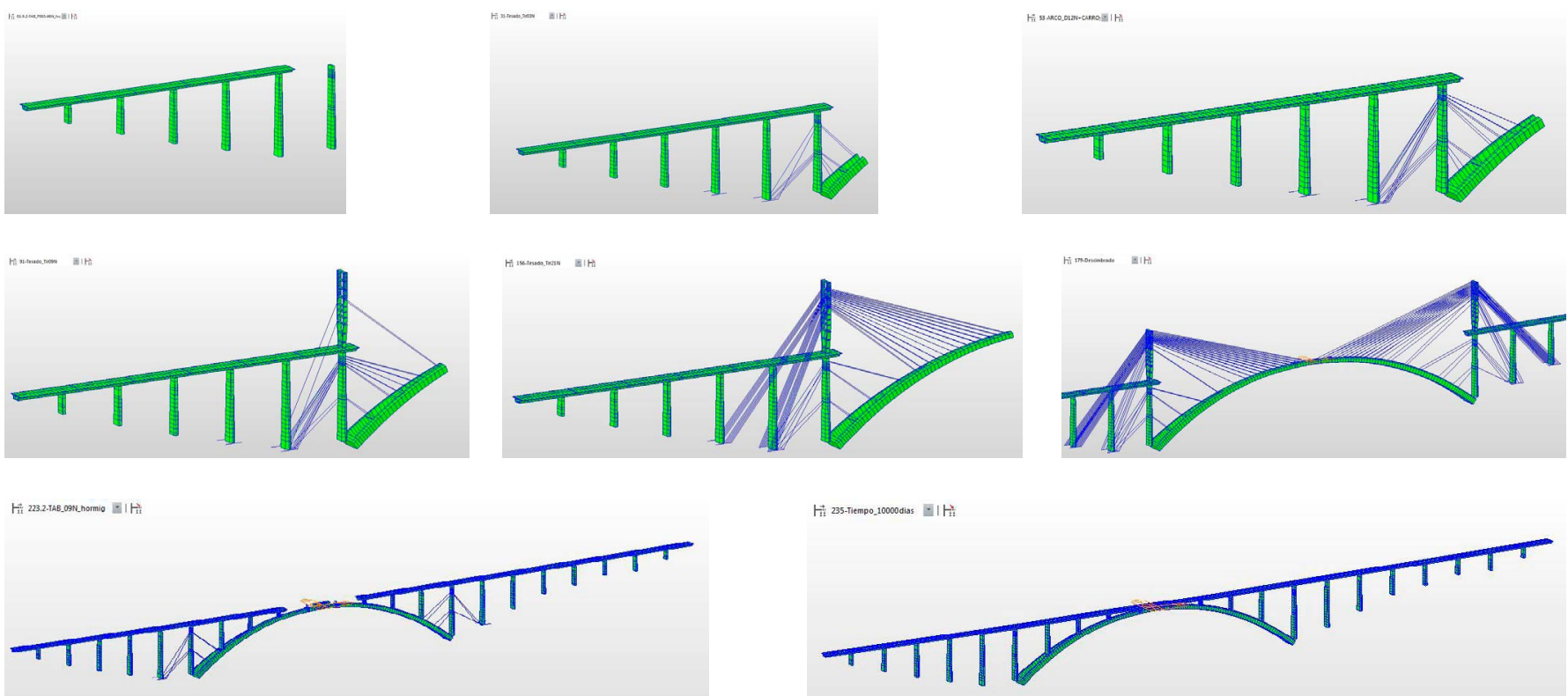

Figura 23. Diferentes etapas del proceso constructivo de la estructura. 


\subsection{Resultados del cálculo durante el proceso constructivo}

A continuación se muestran las tensiones en el voladizo norte del arco durante una sucesión de fases desde la ejecución de

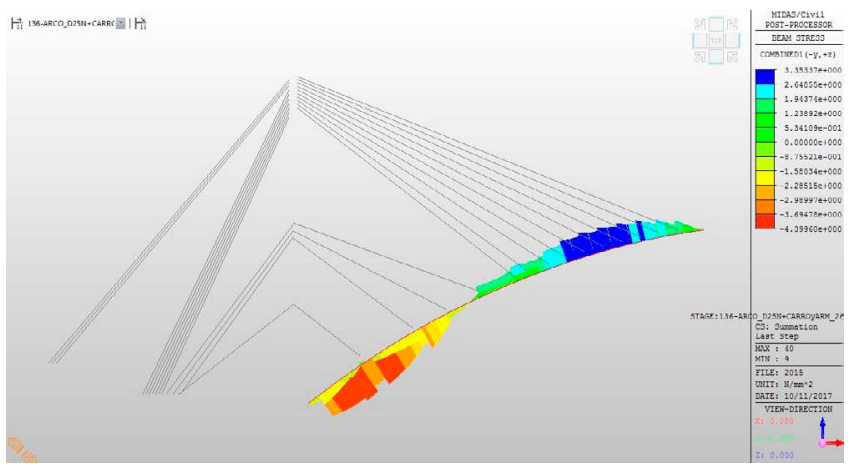

una dovela, destesado de tirantes, tesado de tirantes frontales hasta el avance de carro y ejecución de la siguiente dovela. Se comprueba que las tensiones en fibra superior e inferior son en todas las fases inferiores a $\mathrm{f}_{\mathrm{ctm}}$ (figuras 24, 25, 26 y 27):

Figura 24. Tensiones en fibra superior e inferior cuando el carro de avance se apoya en la dovela $25 \mathrm{~N}$.
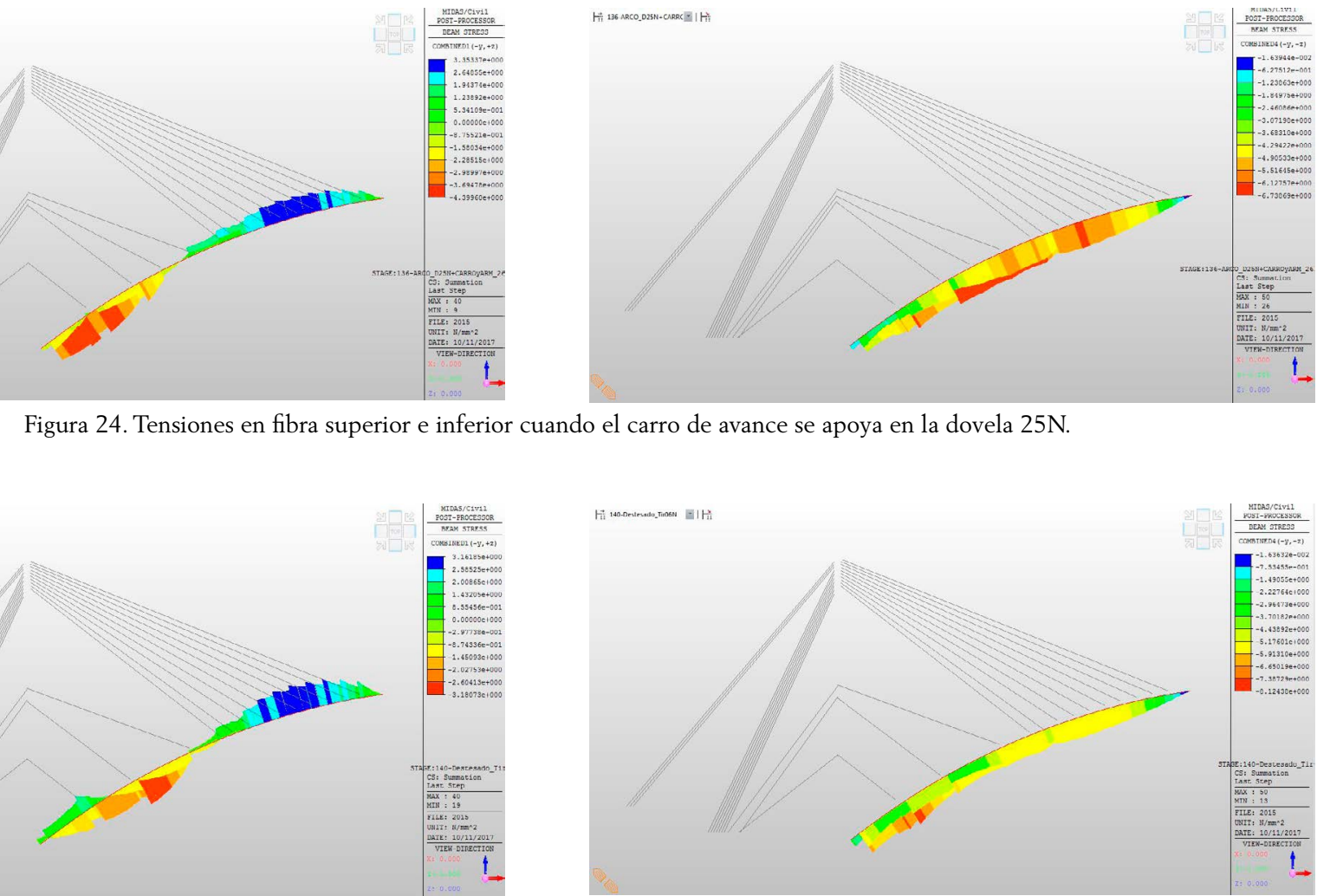

Figura 25. Tensiones en fibra superior e inferior tras el destesado del tirante T06N.
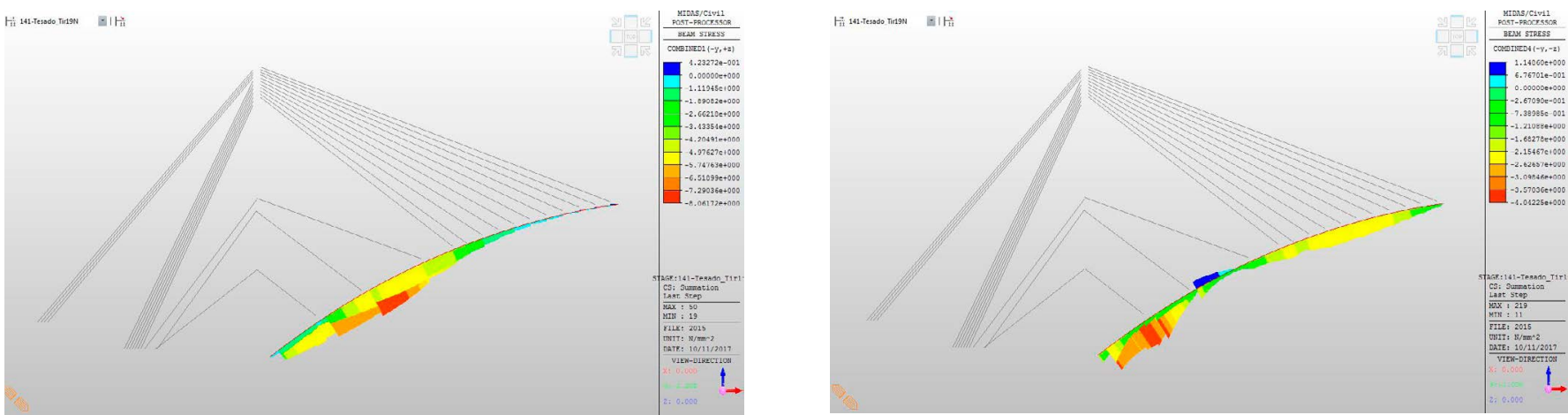

Figura 26. Tensiones en fibra superior e inferior tras el tesado del tirante T19N.
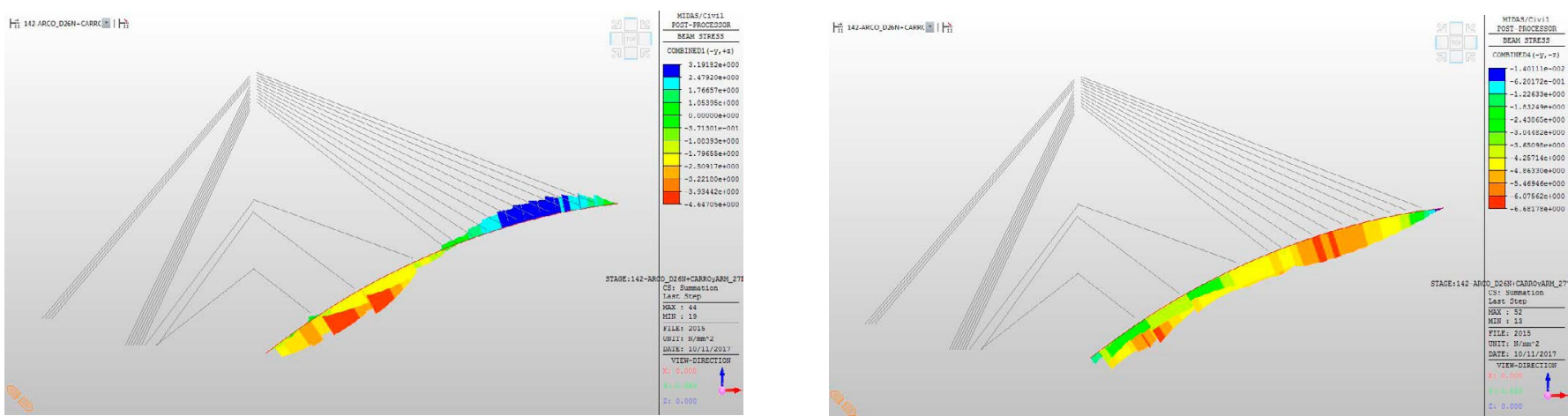

Figura 27. Tensiones en fibra superior e inferior cuando el carro de avance se apoya en la dovela $26 \mathrm{~N}$. 
Las deformaciones verticales acumuladas correspondientes con las fases estudiadas son las siguientes (figuras 28, 29, 30 y 31 ):

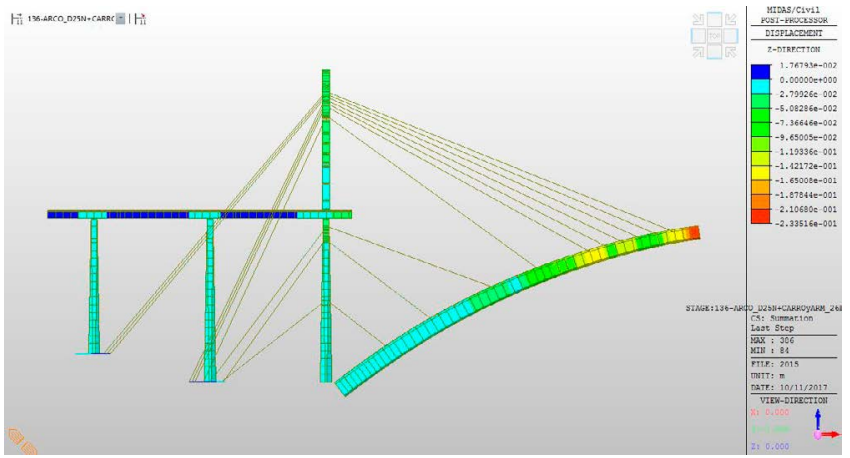

Figura 28. Deformaciones verticales cuando el carro de avance se apoya en la dovela $25 \mathrm{~N}$.

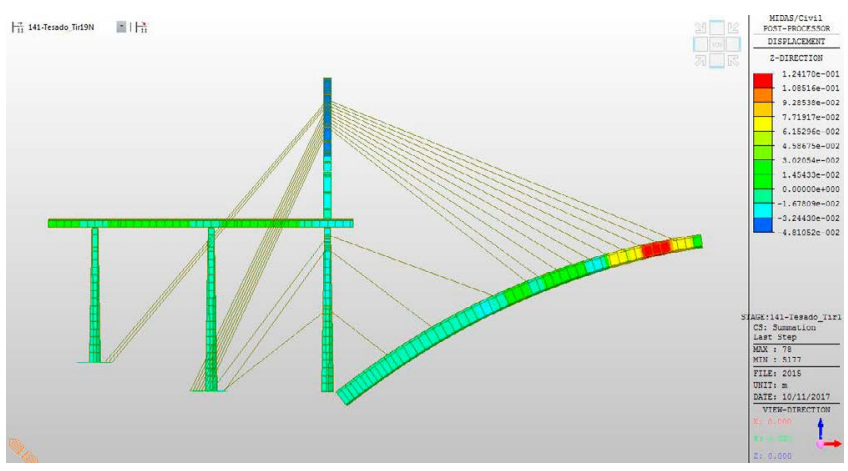

Figura 30. Deformaciones verticales tras el tesado del tirante T19N.

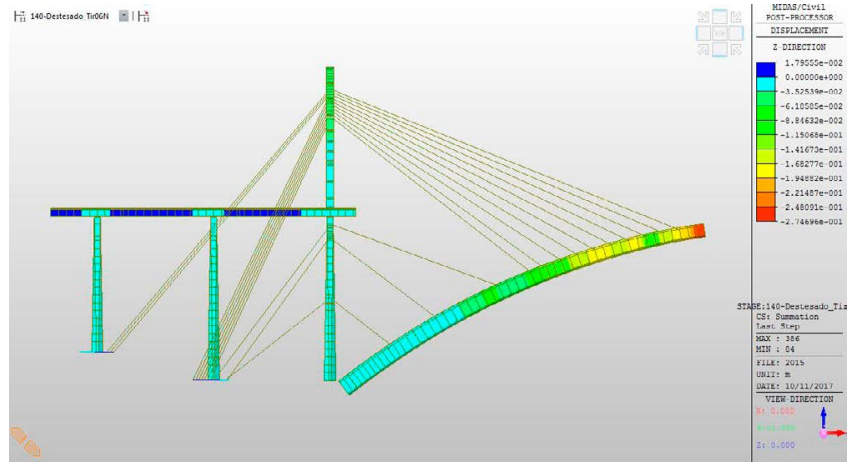

Figura 29. Deformaciones verticales tras el destesado del tirante T06N.

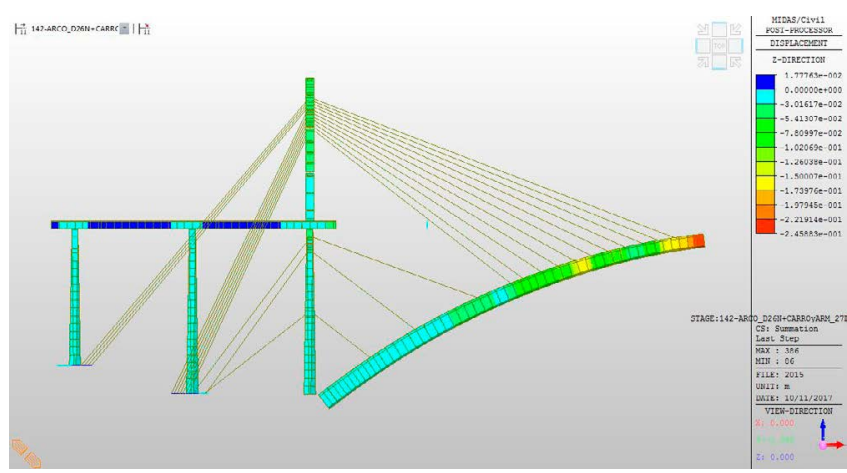

Figura 31. Deformaciones verticales cuando el carro de avance se apoya en la dovela $26 \mathrm{~N}$.

En las siguientes imágenes se recogen los esfuerzos en el arco y en el tablero durante las etapas más significativas del proceso constructivo.

a) Axiles en el arco (figuras 32, 33 y 34)

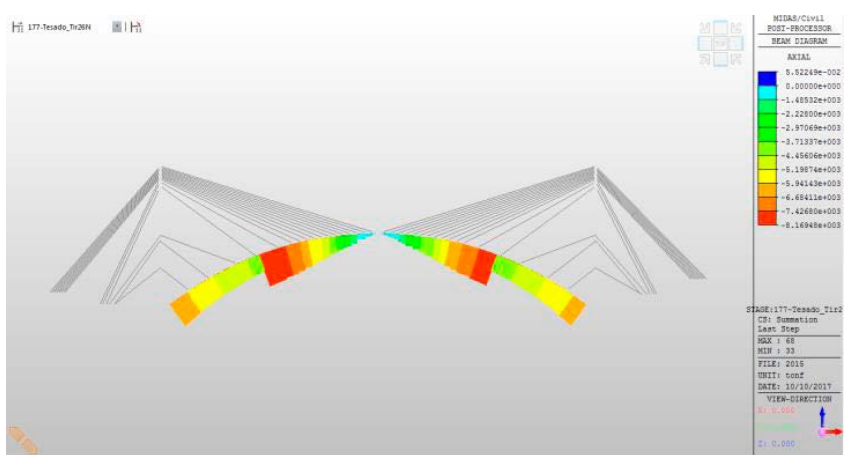

Figura 32. Axiles (tonf) en el arco antes del cierre en clave.

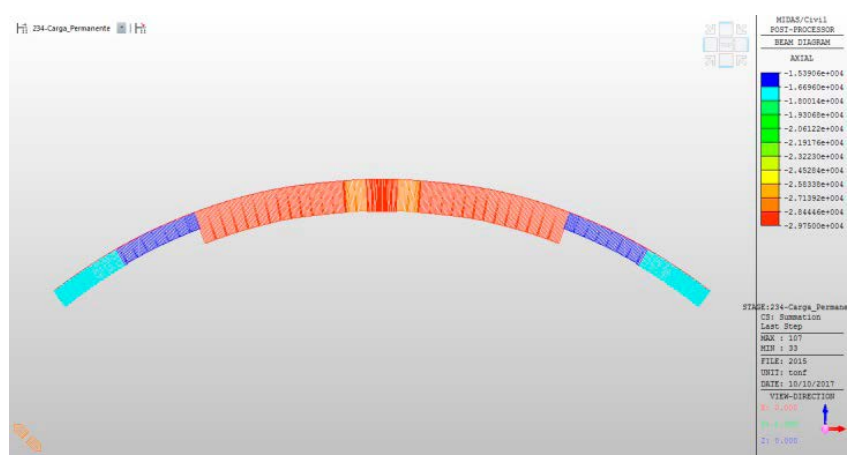

Figura 33. Axiles (tonf) en el arco bajo carga permanente (T0).

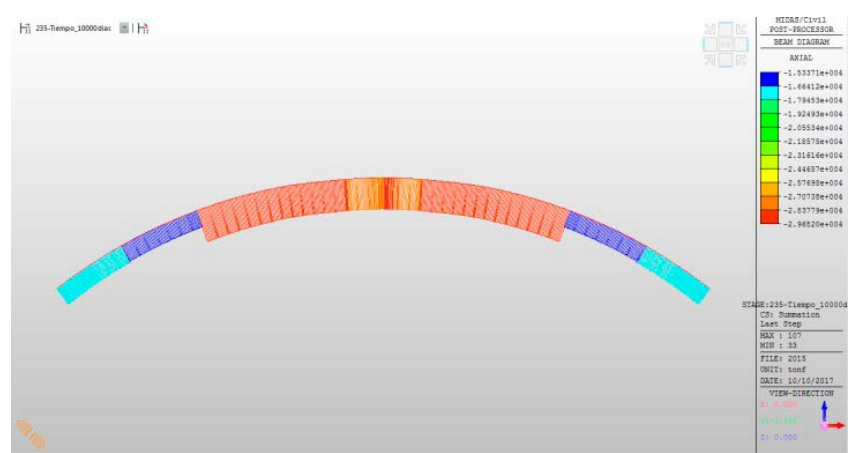

Figura 34. Axiles (tonf) en el arco tras 10000 días (TInf). 


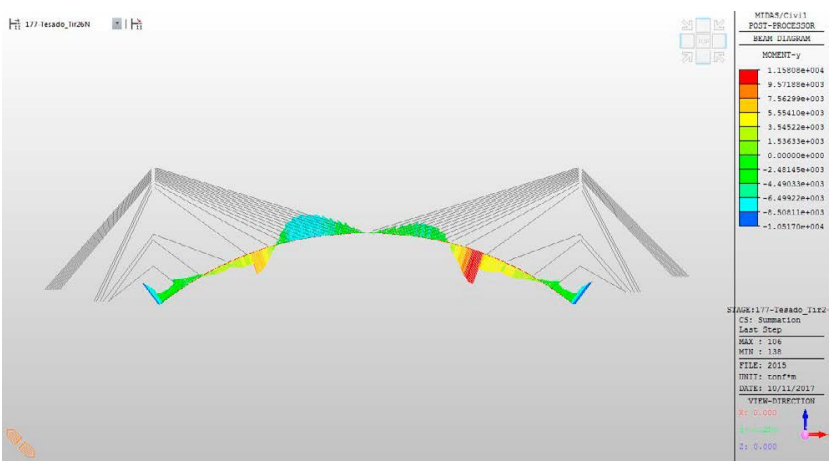

Figura 35. Momentos flectores ( $\mathrm{m} \cdot$ tonf) en el arco antes del cierre en clave.

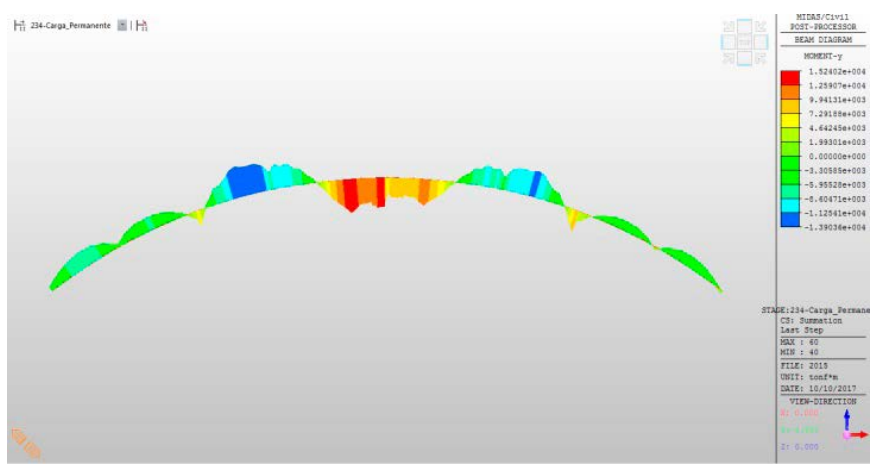

Figura 36. Momentos flectores ( $\mathrm{m} \cdot$ tonf) en el arco bajo carga permanente (T0).

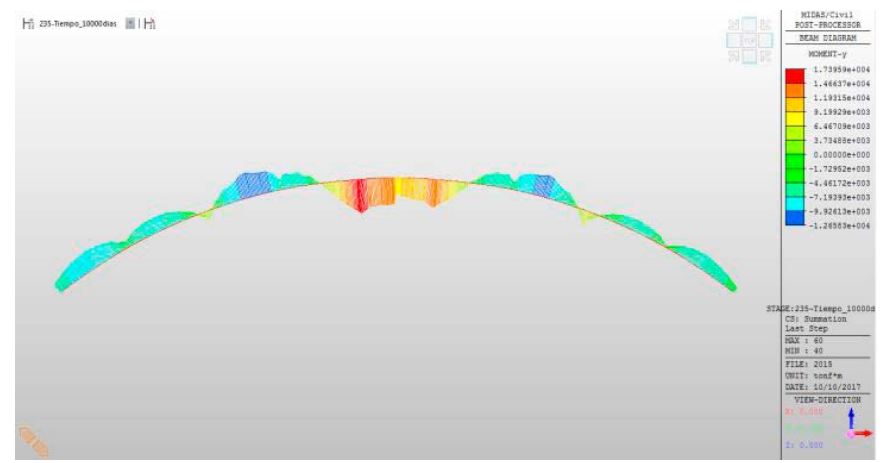

Figura 37. Momentos flectores (m·tonf) en el arco tras 10000 días (TInf).

c) Momentos flectores en tablero (figuras 38 y 39)

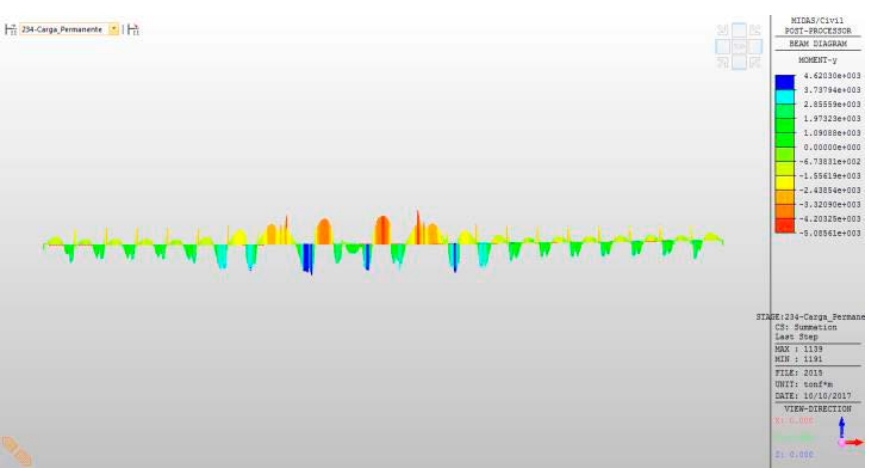

Figura 38. Momentos flectores ( $\mathrm{m} \cdot$ tonf) en el tablero bajo carga permanente (T0).

\subsection{La deformabilidad vertical del tablero}

Un punto determinante de la funcionalidad final de la estructura es el cumplimiento de las consideraciones deformacionales mínimas de la vía para que el tren circule con seguridad y confort. Los límites de deformabilidad recogidas en las IGP y Eurocódigos no resultan fácilmente extrapolables a esta estructura, pues están pensados para dinteles rectos continuos.

Así, los movimientos de origen térmico provocan movimientos de ascenso y descenso de la clave del arco, con una longitud equivalente a una viga continua de 384 metros. Los movimientos debidos a las sobrecargas cuando todo el tablero sobre el arco se encuentra uniformemente cargado son igualmente equivalentes a una viga de 384 metros, y la deformación

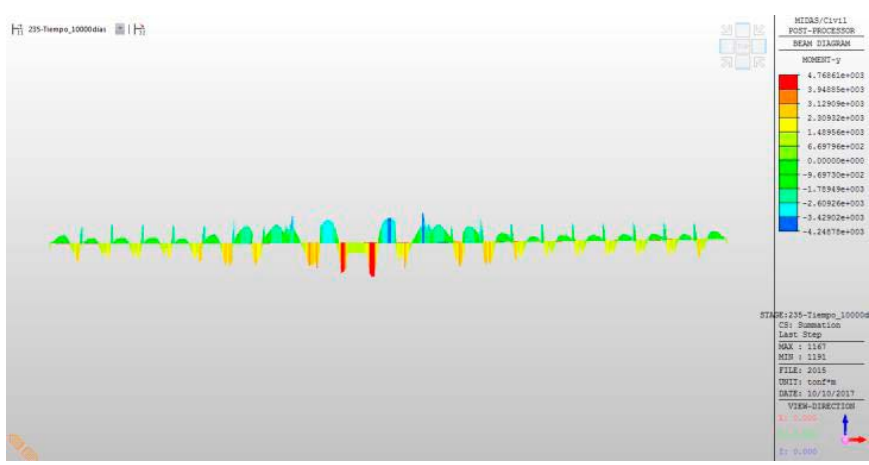

Figura 39. Momentos flectores ( $\mathrm{m} \cdot$ tonf) en el tablero tras 10000 días (Tonf).

conjunta debido a estos dos efectos cumple sobradamente los criterios deformacionales indicados en las IGP. Sin embargo, la deformación provocada por la sobrecarga actuando en una mitad del tablero sobre el arco provoca una deformación antimétrica para una longitud equivalente de aproximadamente $150 \mathrm{~m}$. Si bien esta deformación está también dentro de los límites normativos, es necesario superponerla con la deformación térmica, que como hemos visto tiene una longitud equivalente distinta.

Por todo ello, dada la longitud del vano en relación a la sobrecarga del convoy, se hace necesario realizar comprobaciones adicionales a las recogidas en la normativa, de tal manera que se garantice que los parámetros de trazado resultantes de la geometría deformada son compatibles con los requisitos 


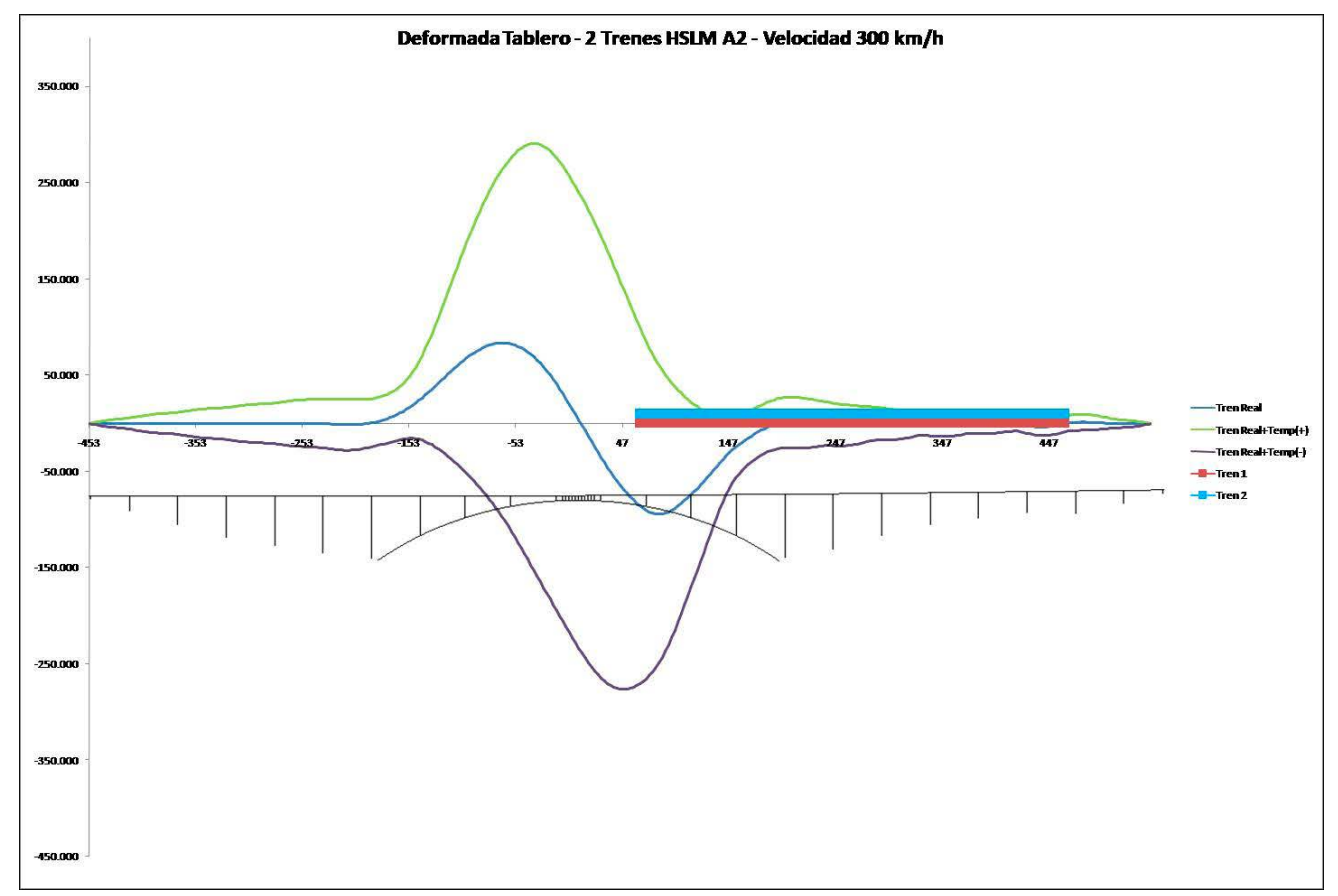

Figura 40. Deformada del tablero para un caso concreto de paso de dos trenes HSLM a $300 \mathrm{~km} / \mathrm{h}$ circulando en paralelo.

de trazado de las líneas de alta velocidad. Para ello, se realizó un cálculo dinámico de todos los trenes posibles para sus velocidades de circulación y la corrección del parámetro del acuerdo vertical $\left(\mathrm{K}_{\mathrm{v}}\right)$ de acuerdo con la deformada resultante, obteniéndose así la rama de la parábola de pendiente máxima en la posición del tren en la cual la flecha resulta máxima para cada tren y velocidad (figura 40).

Estos pasos de trenes por el puente se estudiaron con todos los escenarios posibles para barrer cualquier posibilidad de organización de la circulación incluyendo los efectos térmicos:

- Un tren circulando en una dirección.

- Dos trenes circulando en paralelo con la misma dirección y velocidad.

- Dos trenes circulando en direcciones opuestas con el cruce en la posición más desfavorable.

Los cálculos realizados permiten obtener la deformación de la estructura bajo el paso de los trenes reales a distintas velocidades de paso, incluyendo los efectos dinámicos, y compararlo con los requisitos de trazado, que es función también de la velocidad de paso del tren.

Resulta importante señalar que los resultados obtenidos demuestran que el criterio básico de diseño del arco de evitar la fisuración en situaciones de servicio, tanto en fase de construcción como en explotación, resulta fundamental no solo para el cumplimiento de los distintos ELU sino los criterios de deformación (debido a que la fisuración flexibiliza la estructura y aumenta por tanto la deformación y los efectos de segundo orden).

\subsection{Cálculo dinámico}

Los fenómenos dinámicos originados por el tráfico ferroviario se deben, fundamentalmente, a las cargas verticales móviles transmitidas por las ruedas de los vehículos. La variación del punto de aplicación de estas cargas origina una excitación dinámica de la estructura. A ello hay que añadir los efectos ocasionados por las irregularidades de carril y ruedas. Por otra parte, generalmente estas cargas se encuentran a unos espaciamientos determinados a lo largo del tren, lo que da lugar a excitaciones periódicas que pueden producir resonancia en la estructura. Los efectos dinámicos suponen un incremento respecto a la respuesta estática en las deformaciones y esfuerzos soportados por la estructura. Adicionalmente, pueden generar desplazamientos o aceleraciones que produzcan efectos nocivos en la estructura o en el balasto, o que comprometan la seguridad de la circulación, o incomodidad en el viajero.

El viaducto de Almonte requiere un cálculo dinámico basado en la integración directa en el tiempo con cargas móviles. Para ello, es necesario realizar los cálculos que resulten envolventes de los 10 trenes dinámicos universales $\mathrm{A}$, efectuando un barrido desde la velocidad mínima de $20 \mathrm{~km} / \mathrm{h}$, que puede considerarse como velocidad cuasiestática, hasta la velocidad máxima previsible, $\mathrm{V}_{\max }=1 \cdot 2 \cdot \mathrm{V}$, donde $\mathrm{V}$ es la velocidad máxima permitida (de proyecto, $350 \mathrm{~km} / \mathrm{h}$ ) en la línea. El incremento de velocidades entre los distintos cálculos es de $10 \mathrm{~km} / \mathrm{h}$.

Habitualmente se considera que existe una relación lineal entre los desplazamientos y las solicitaciones producidas en el puente. Sin embargo, en este caso, esta relación sí se da en el arco, pero no en el tablero. Dado que el arco constituye un apoyo elástico para el tablero, no existe proporcionalidad entre solicitaciones y flechas, ya que las flechas en el tablero provienen de la suma de dos componentes: las debidas a la propia deformabilidad del tablero y las debidas a la deformación del arco sobre el que se apoya.

Por tanto, el coeficiente de impacto con el que se calculó el tablero, no se obtuvo a partir de la relación de deformaciones producidas por los trenes, como en las estructuras convencionales, sino que se determinó a partir de los esfuerzos dinámicos. Además, como resultado del cálculo dinámico se 


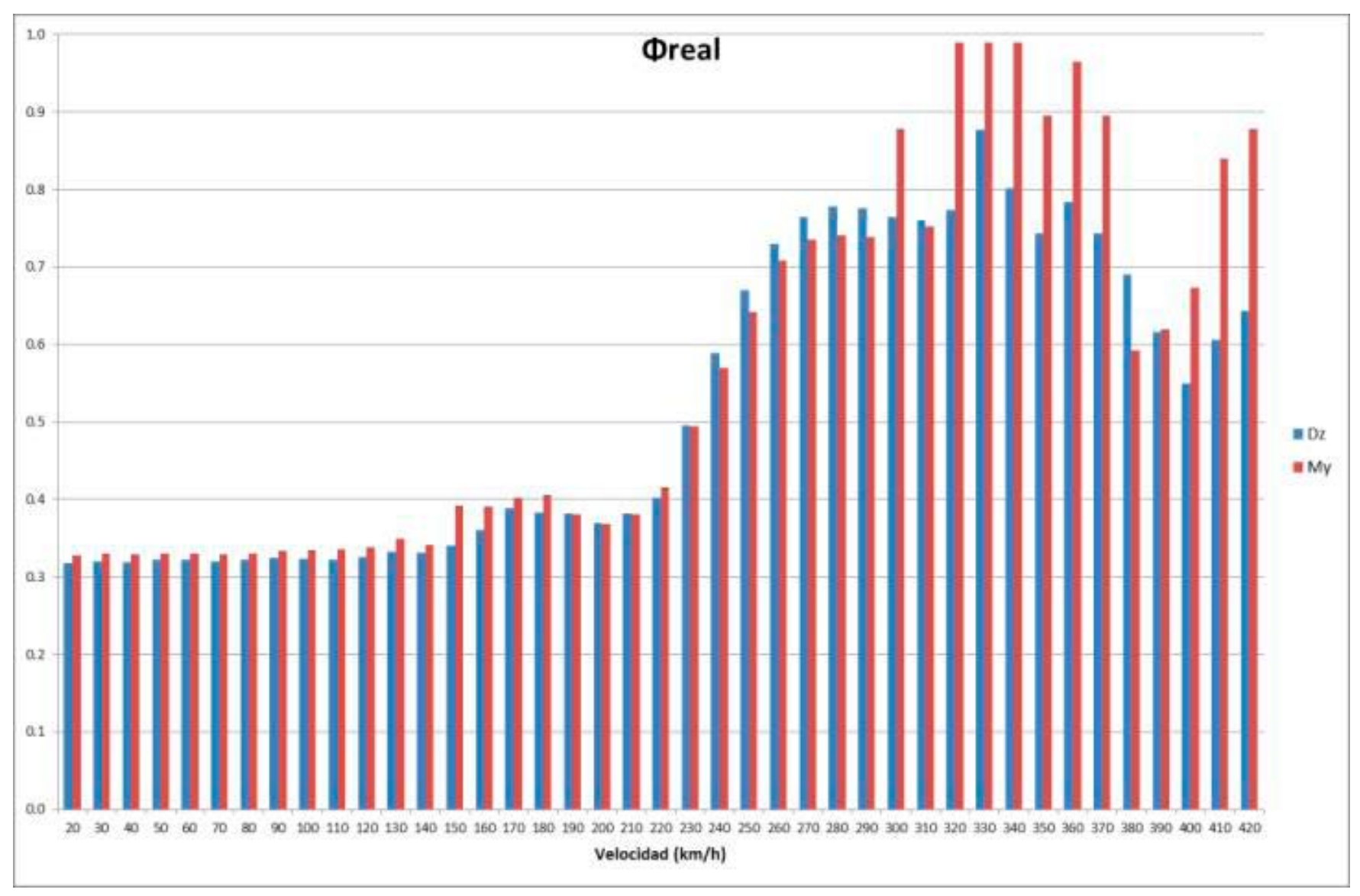

Figura 41. Coeficientes de impacto en tablero para las diferentes velocidades.

obtuvieron las aceleraciones que permiten verificar los estados límite de servicio.

\subsubsection{Coeficiente de impacto en tablero y arco}

En la siguiente figura se muestra el coeficiente de impacto (relación entre envolvente de HSLM-A y UIC-71) obtenido en el tablero tanto para desplazamientos como para momento flector, observando, que como se ha descrito anteriormente, estos coeficientes no coinciden cuando los efectos dinámicos son relevantes (figura 41).

Los coeficientes obtenidos muestran que aunque los esfuerzos bajo el tren real están cubiertos con los obtenidos estáticamente por el tren tipo LM71, resultan más elevados que los obtenidos normalmente en los tableros de estructuras convencionales de alta velocidad. En este caso se aprecia que para altas velocidades, los esfuerzos en el tablero bajo el paso de los trenes reales amplificados dinámicamente se asemejan en algunas secciones a los valores del tren tipo LM71 con el cual se dimensionan estáticamente las estructuras.

A continuación se muestra la comparativa de los momentos flectores producidos en el tablero por el paso tanto estático como dinámico de los trenes HSLM-A y el paso estático del tren UIC-71, comprobando que los esfuerzos obtenidos por el cálculo dinámico son siempre inferiores a los que genera el tren UIC-71 en el caso estático (figura 42):

El factor de amplificación dinámico resulta máximo en los vanos laterales inmediatamente anteriores a los vanos del tablero que ya se encuentran sobre el arco.
En la siguiente gráfica se lleva a cabo la comparativa de los momentos flectores que producen los diferentes trenes en el arco, obteniendo un resultado análogo al del tablero (figura 43).

\subsubsection{Flechas y aceleraciones}

Se muestran a continuación la evolución de las flechas y aceleraciones con el tiempo en algunos puntos significativos para la velocidad de $330 \mathrm{~km} / \mathrm{h}$ (por ser esta la velocidad a la que se producen los mayores efectos dinámicos), comprobando que las aceleraciones obtenidas son siempre inferiores a $0.35 \mathrm{~g}$ (figuras 44, 45, 46, 47, 48 y 49):

Los resultados demuestran el buen comportamiento dinámico del viaducto, debido fundamentalmente al empleo de hormigón en toda la estructura, que conlleva a una mejor respuesta ante fenómenos vibratorios respecto a otras soluciones, debido a su masa y amortiguamiento.

\section{5.}

\section{CONCLUSIONES}

Es adecuado señalar que los nuevos materiales de altas prestaciones, las modernas herramientas de cálculo y modelización aeroelástica y los tratamientos semiprobabilísticos normativos de los formatos de seguridad han permitido diseñar el puente sobre el río Almonte, y que el uso conjunto e intensivo de todos ellos ayudan a superar los rangos de luz 


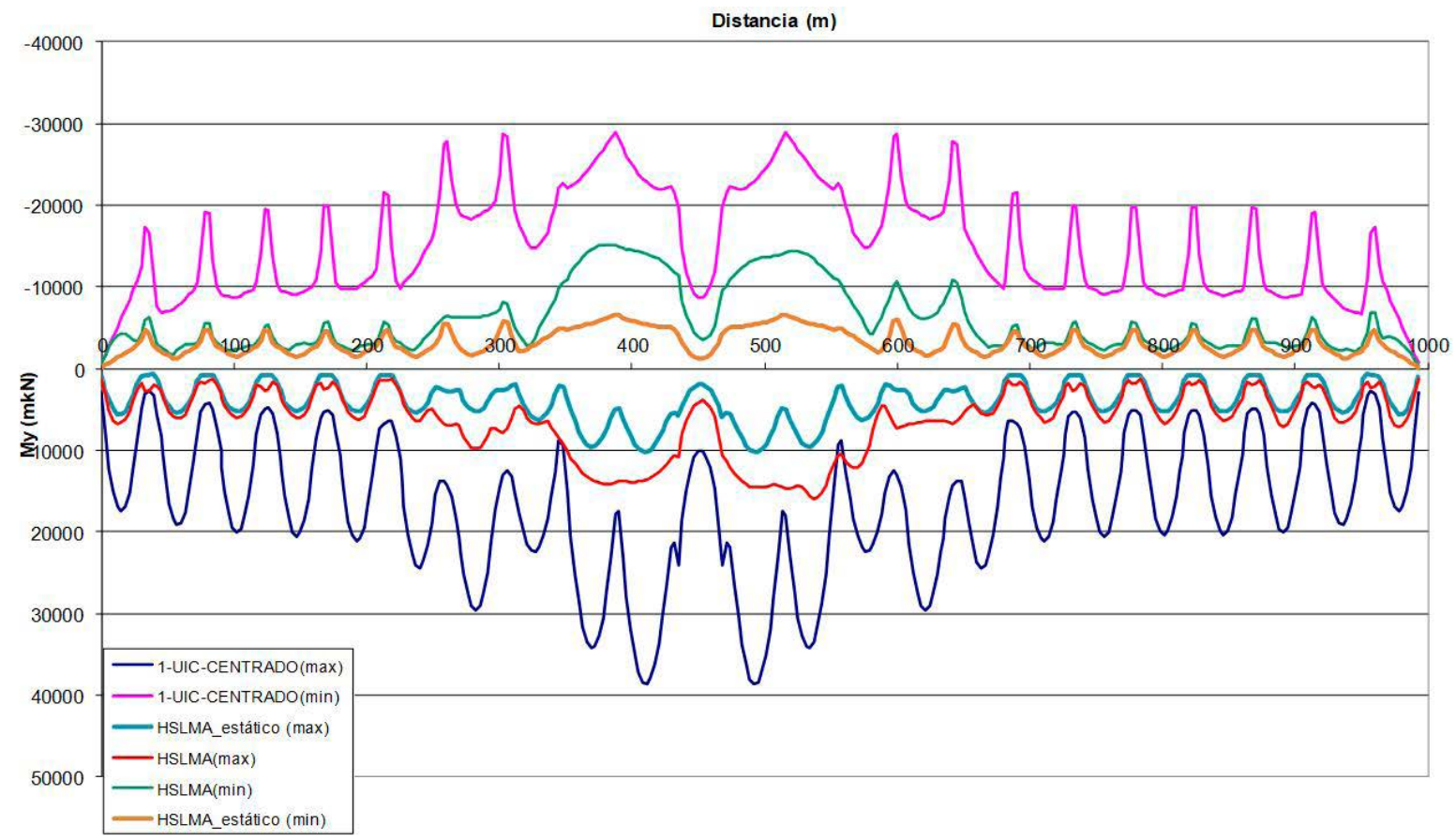

Figura 42. Momentos flectores debidos a los diferentes trenes en el tablero.

ENVOLVENTE TRENES UNIVERSALES - VELOCIDAD $330 \mathrm{~km} / \mathrm{h}$

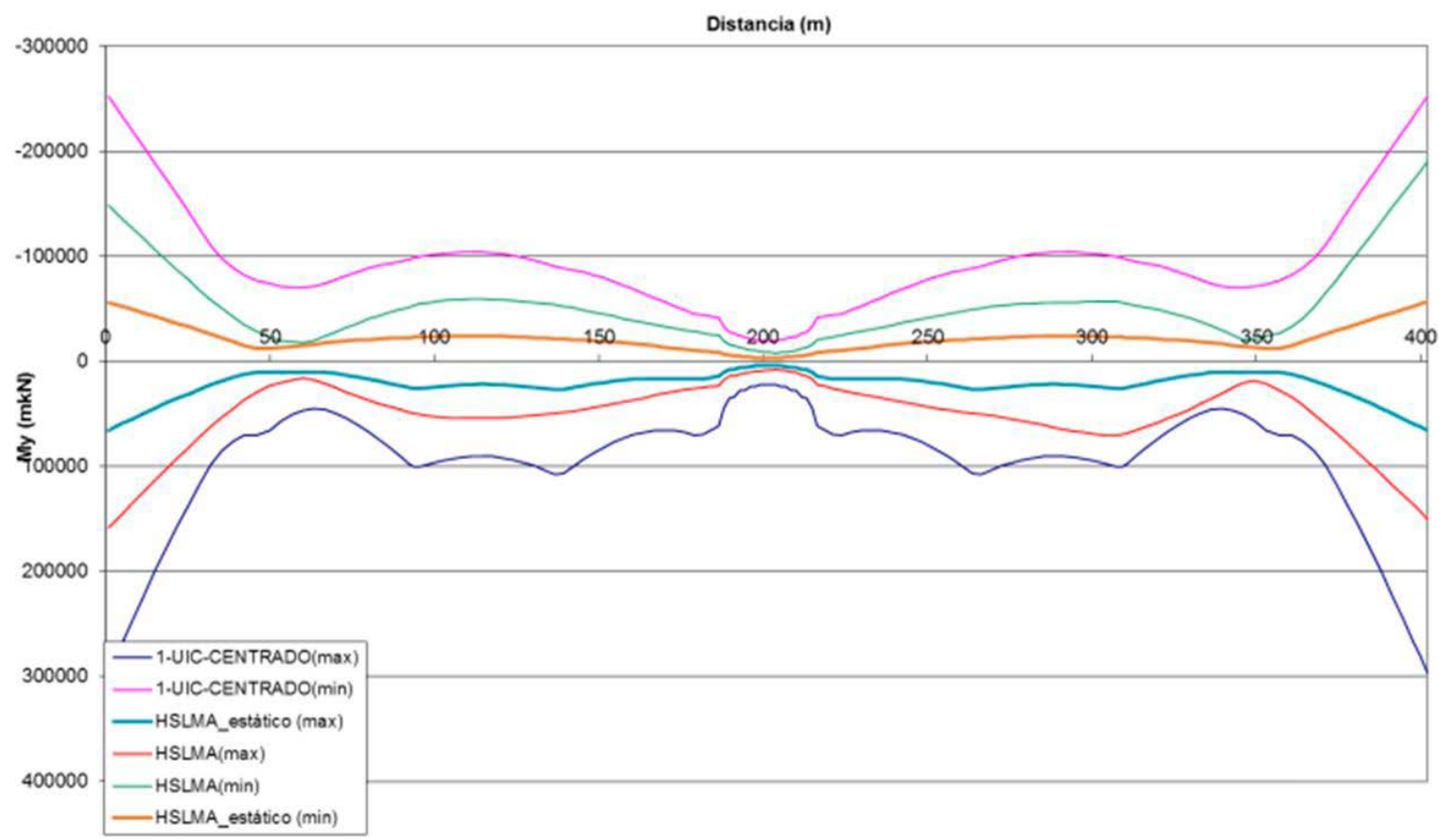

Figura 43. Momentos flectores debidos a los diferentes trenes en el arco.

de utilización que hasta ahora se habían establecido para los puentes ferroviarios.

Al hacer uso de estas herramientas se ha podido refinar el criterio de encaje de la directriz del arco para tener en cuenta la influencia de la amplificación dinámica de las sobrecargas ferroviarias para Altas Velocidades, y se puede concluir que el análisis no lineal en geometría y material es el único que permite valorar con precisión los niveles de seguridad con que se dimensiona realmente la estructura, dada su luz, esbeltez y la importancia de las sobrecargas sobre el total de las cargas.

\section{Referencias}

[1] TORRES, J.; SANTAMARÍA, A.J.; ARENAS, J.J.; DIAZ DEL VALLE, J.; LORENZO, D. "Tipología. Arcos. Funicularidad". Servicio de Publicaciones de la E.T.S. de Ingenieros de Caminos, Canales y Puertos. Universidad de Cantabria. ISBN: 84-86928-14-1.

[2] CAPELLAN, G.; BEADE, H.; ARENAS, J.J.; GARCIA, P.; MEANA, I. “Di- 


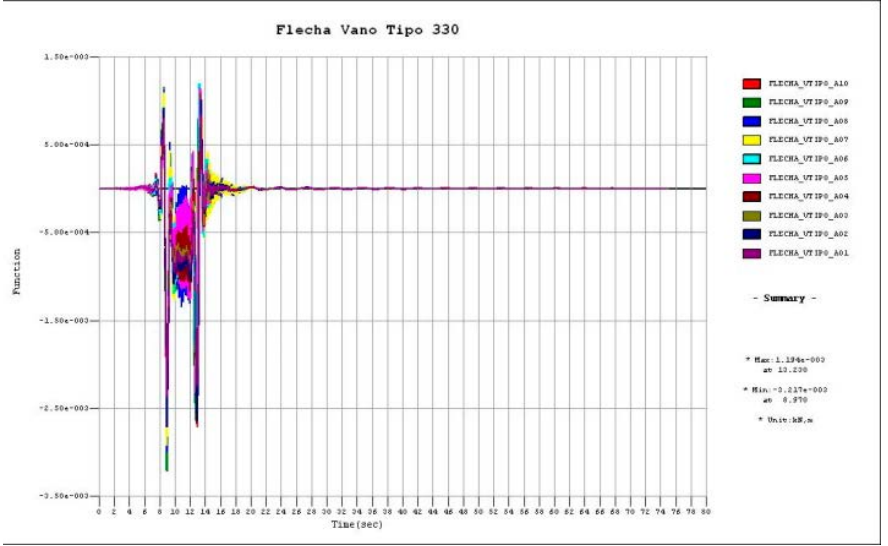

Figura 44. Evolución de las flechas en el tiempo para el centro de vano tipo.

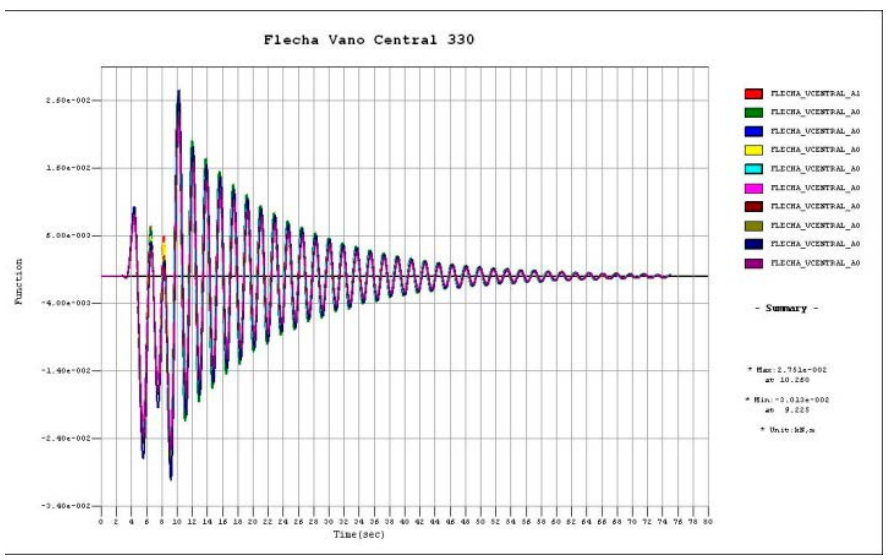

Figura 46. Evolución de las flechas en el tiempo para el centro de vano sobre el arco (P10-P11).

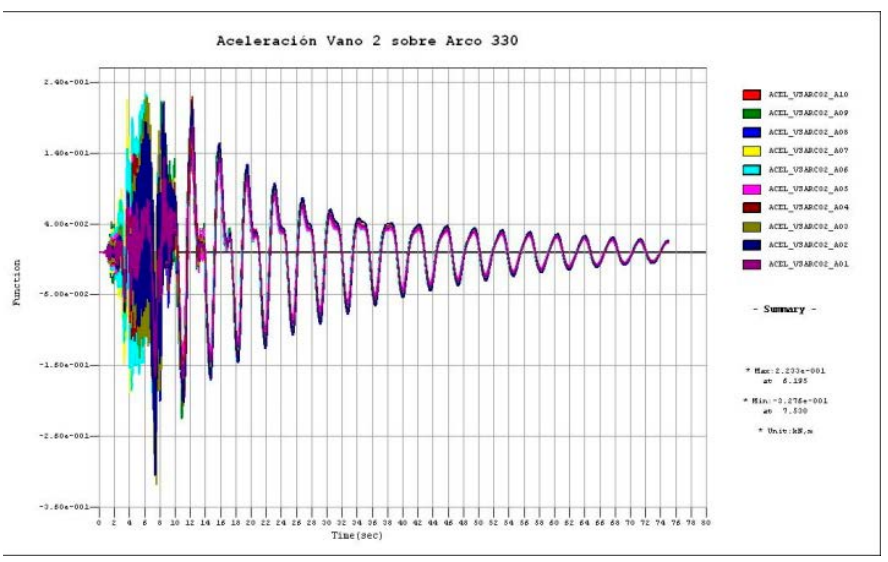

Figura 48. Evolución de las aceleraciones en el tiempo para el centro de vano 2 sobre el arco (P7-P8).

seño del puente arco de Alta Velocidad sobre el río Almonte en el Embalse de Alcántara". VI Congreso Internacional de Estructuras de la ACHE. Asociación Científico-técnica del Hormigón Estructural. 2014. ISBN: 978-8489670-80-8

[3] ARENAS, J.J; CAPELLAN, G.; BEADE, H.; MEANA, I. "Viaducto sobre el río Almonte en el Embalse de Alcántara para la Línea Ferroviaria de Alta Velocidad Madrid - Extremadura. Talayuela - Cáceres". V Congreso Internacional de Estructuras de la ACHE. Asociación Científico-técnica del Hormigón Estructural 2011. ISBN: 978-84-89670-73-0

[4] ARENAS, J.J.; HACAR, F.; GARCIA-ARANGO, I.; GONZALEZ, M.; GURRIARÁN, R.; PANTALEÓN. "Carretera Nacional 632 de Ribadesella a

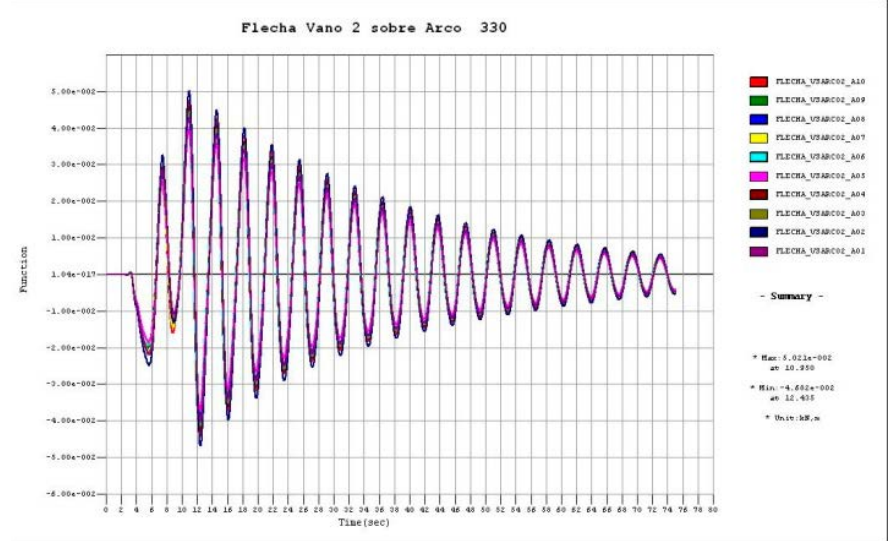

Figura 45. Evolución de las flechas en el tiempo para el centro de vano 2 sobre el arco (P7-P8).

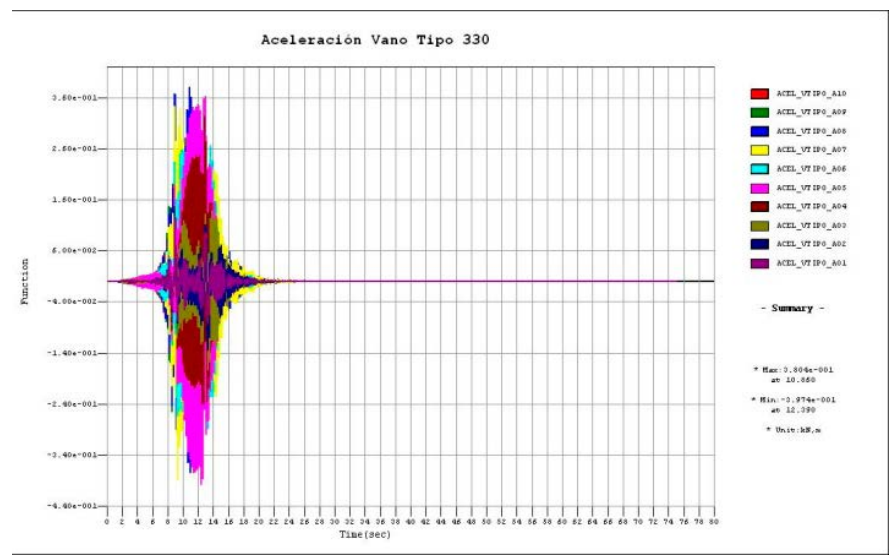

Figura 47. Evolución de las aceleraciones en el tiempo para el centro de vano tipo.

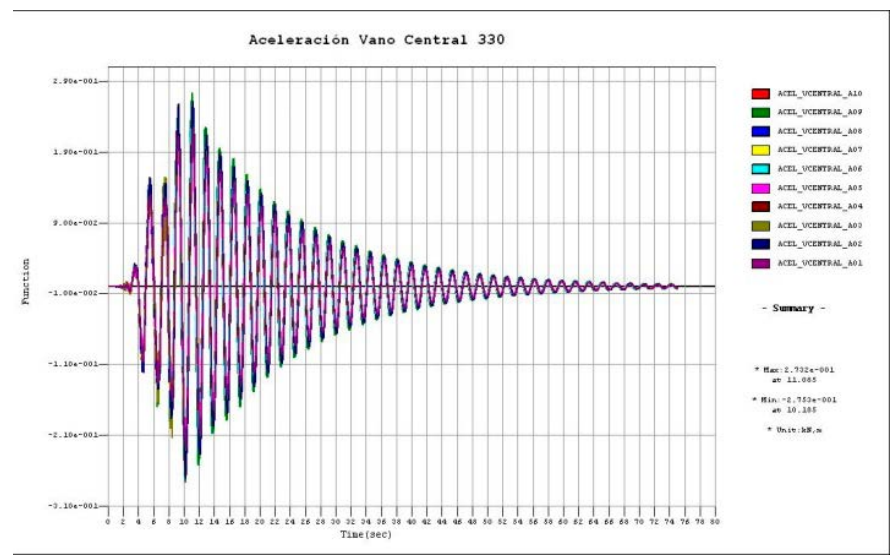

Figura 49. Evolución de las aceleraciones en el tiempo para el centro de vano sobre el arco (P10-P11).

Luarca. Tramo: Novellana - Cadavedo; Arco de la Regenta, Viaducto Pintor Fierros". Revista de Obras Públicas, 1997, n³363, Marzo, p.17-43.

[5] EN 1992-2:2005, EUROCÓDIGO 2 (2005): Proyecto de estructuras de hormigón - Parte 2: Puentes de hormigón - Cálculo y disposiciones constructivas - Apartado 5.7 Cálculo no lineal y Anexo PP: Formato de seguridad para el cálculo no lineal.

[6] DESIGNERS' GUIDE TO EN 1992, Eurocode 2: Design of concrete structures - Part 2: Concrete Bridges. Eurocode Designers' Guide Series. 